\title{
Derived equivalences of canonical covers of hyperelliptic and Enriques surfaces in positive characteristic
}

\author{
Katrina Honigs ${ }^{1}$ - Luigi Lombardi ${ }^{2}$. Sofia Tirabassi ${ }^{3,4}$
}

Received: 29 November 2016 / Accepted: 25 June 2019 / Published online: 1 August 2019

(c) The Author(s) 2019

\begin{abstract}
We prove that any Fourier-Mukai partner of an abelian surface over an algebraically closed field of positive characteristic is isomorphic to a moduli space of Gieseker-stable sheaves. We apply this fact to show that the set of Fourier-Mukai partners of a canonical cover of a hyperelliptic or Enriques surface over an algebraically closed field of characteristic greater than three is trivial. These results extend earlier results of Bridgeland-Maciocia and Sosna to positive characteristic.
\end{abstract}

\section{Introduction}

The main motivation of this paper is the recent series of results in the study of equivalences of derived categories of sheaves of smooth projective varieties over fields other than the field of complex numbers. For instance, over finite fields, the first named author proves that the Hasse-Weil zeta function of an abelian variety, as well as of smooth varieties of dimension at most three, is unaltered under equivalences of derived categories [9,11]. Moreover, Ward in his thesis [35] produces examples of genus one curves over $\mathbf{Q}$ admitting an arbitrary number of distinct Fourier-Mukai partners, revealing in this way consistent differences with the case of elliptic curves over $\mathbf{C}$. Finally, Lieblich and Olsson in [17] extend to positive characteristic seminal works of Mukai and Orlov concerning derived equivalences of $K 3$ surfaces. In particular, they prove that any Fourier-Mukai partner of a $K 3$ surface $X$ over an algebraically closed field of characteristic $p \neq 2$ is a moduli space of Gieseker-stable

\footnotetext{
Sofia Tirabassi

sofia.tirabassi@uib.no; tirabassi@math.su.se

Katrina Honigs

khonigs@uoregon.edu

Luigi Lombardi

luigi.lombardi@unimi.it

1 Department of Mathematics, Fenton Hall, University of Oregon, Eugene, OR 97403-1222, USA

2 Department of Mathematics, University of Milan, Via Cesare Saldini 50, Milan 20133, Italy

3 Department of Mathematics, Stockholm University, 10691 Stockholm, Sweden

4 Department of Mathematics, University of Bergen, Allègaten 52, Bergen, Norway
} 
sheaves on $X$, and in addition $X$ admits only a finite number of Fourier-Mukai partners. While Orlov's proof relies on Hodge theory, Lieblich-Olsson's proof relies on deformation theory of perfect complexes and on the theory of liftings to the Witt ring.

Inspired by the results of [17], in this paper we focus on special classes of abelian and $K 3$ surfaces that arise as canonical covers of hyperelliptic and Enriques surfaces. Our first main result is an extension of a work of Sosna [34, Theorem 1.1] to positive characteristic.

Theorem 1.1 Let $S$ be a hyperelliptic surface over an algebraically closed field of characteristic $p>3$ and let $A$ be its canonical cover. Then any smooth projective surface that is derived equivalent to $A$ is isomorphic to either $A$ or its dual $\widehat{A}$.

We refer to Theorem 4.4 for a slightly stronger result. One of the main ingredients in the proof of Theorem 1.1 is the characterization of Fourier-Mukai partners of abelian surfaces in positive characteristic as moduli spaces of Gieseker-stable sheaves. In particular we extend the result [6, Theorem 5.1] of Bridgeland-Maciocia to positive characteristic. In the following we denote by $\mathbf{D}(X)$ the bounded derived category of coherent sheaves on a smooth projective variety $X$.

Theorem 1.2 Let $A$ be an abelian surface over an algebraically closed field $k$ of positive characteristic and let $Y$ be a smooth projective variety over $k$. Suppose furthermore that there is an equivalence of triangulated categories $\Phi: \mathbf{D}(A) \rightarrow \mathbf{D}(Y)$. Then $Y$ is an abelian surface and $A$ is isomorphic to a moduli space of Gieseker-stable sheaves on either $Y$ or its dual $\widehat{Y}$.

The proof of the previous theorem is based on the notion of filtered equivalence ( $c f$. $[17,18])$. We recall that a derived equivalence $\Phi: \mathbf{D}(X) \rightarrow \mathbf{D}(Y)$ of surfaces induces a homomorphism $\Phi^{\mathrm{CH}}: \mathrm{CH}^{*}(X) \rightarrow \mathrm{CH}^{*}(Y)$ between the numerical Chow groups. Then one says that $\Phi$ is filtered if $\Phi^{\mathrm{CH}}(0,0,1)=(0,0,1)$. In Proposition 3.1 we show that in the case of abelian surfaces, a filtered equivalence induces an isomorphism between the surfaces. Hence in order to complete the proof of Theorem 1.2, we construct an equivalence of derived categories

$$
\Xi: \mathbf{D}(A) \stackrel{\Phi}{\longrightarrow} \mathbf{D}(Y) \stackrel{\Psi}{\longrightarrow} \mathbf{D}(Y)
$$

where $\Psi$ is an autoequivalence such that the moduli space $\mathcal{M}_{Y}(v)$ of Gieseker-stable sheaves with $v=\Xi^{\mathrm{CH}}(0,0,1)$ is a smooth surface that admits a universal family $\mathcal{U}$. This completes the proof as the composition of $\Xi$ with the Fourier-Mukai functor associated to $\mathcal{U}$ is a filtered equivalence. We have been able to perform this strategy only if the rank component $r$ of $\Phi^{\mathrm{CH}}(0,0,1)$ is non-zero. In the other case $r=0$, we had to involve Mukai's equivalence $\mathcal{S}_{Y}: \mathbf{D}(Y) \rightarrow \mathbf{D}(\widehat{Y})$ induced by the Poincaré bundle in order to apply the same plan. This explains why the conclusions of Theorem 1.2 and [6, Theorem 5.1] are not completely symmetric.

In Sect. 6 we observe that one can push the techniques of [17] a little further in order to prove that $K 3$ surfaces that are canonical covers of Enriques surfaces in characteristic $p>3$ do not admit any non-trivial Fourier-Mukai partner. This in particular extends the second part of the result of Sosna [34, Theorem 1.1] to positive characteristic.

Theorem 1.3 Let $S$ be an Enriques surface over an algebraically closed field of characteristic $p>3$ and let $X$ be its canonical cover. Then any smooth projective surface that is derived equivalent to $X$ is isomorphic to $X$. 


\subsection{Notation}

Unless otherwise specified we work over an algebraically closed field $k$ of positive characteristic $p$.

\section{Background material}

\subsection{Fourier-Mukai transforms and Chow groups}

Let $k$ be an algebraically closed field of positive characteristic $p$. The bounded derived category of sheaves of a smooth projective variety $X$ is defined as $\mathbf{D}(X):=D^{b}(\mathcal{C} \operatorname{oh}(X))$. The category $\mathbf{D}(X)$ is $k$-linear and triangulated. If $Y$ is another smooth projective variety, an object $\mathcal{E}$ in $\mathbf{D}(X \times Y)$ defines a Fourier-Mukai functor via the assignment:

$$
\Phi_{\mathcal{E}}: \mathbf{D}(X) \rightarrow \mathbf{D}(Y), \quad \mathcal{F} \mapsto \mathbf{R} p_{2 *}\left(p_{1}^{*} \mathcal{F} \stackrel{\mathbf{L}}{\otimes} \mathcal{E}\right)
$$

where $p_{1}$ and $p_{2}$ denote the projections from $X \times Y$ onto the first and second factor respectively. An important theorem of Orlov tells us that any equivalence $F: \mathbf{D}(X) \rightarrow \mathbf{D}(Y)$ is of Fourier-Mukai type, i.e. there exists a unique up to isomorphism kernel $\mathcal{E}$ in $\mathbf{D}(X \times Y)$ such that $F \simeq \Phi_{\mathcal{E}}$. Finally we recall that the composition of Fourier-Mukai transforms is again of Fourier-Mukai type.

Now consider an abelian surface $A$ over $k$. We denote by $\mathrm{CH}^{*}(A)_{\text {num }}=\oplus_{i} \mathrm{CH}^{i}(A)_{\text {num }}$ the graded ring of algebraic cycles modulo numerical equivalence so that

$$
\mathrm{CH}^{0}(A)_{\text {num }} \simeq \mathbf{Z}, \quad \mathrm{CH}^{1}(A)_{\text {num }} \simeq \mathrm{NS}(A) \text { and } \mathrm{CH}^{2}(A)_{\text {num }} \simeq \mathbf{Z},
$$

where $\mathrm{NS}(A)$ denotes the Néron-Severi group of $A$ up to torsion. Moreover we set $\mathrm{CH}^{*}(A)_{\text {num }, \mathbf{Q}}:=\mathrm{CH}^{*}(A)_{\text {num }} \otimes \mathbf{Q}$. For an object $\mathcal{F}$ in $\mathbf{D}(A)$ we denote by $v(\mathcal{F}) \in$ $\mathrm{CH}^{*}(A)_{\text {num }, \mathbf{Q}}$ its Mukai vector (see [7, Sect. 5.2] and [2, p. 3]). Hence the Mukai vector of a locally free sheaf $E$ on $A$ is $v(E)=\left(\operatorname{rk}(E), c_{1}(E), \chi(E)\right)$ and the map

$$
v: \mathbf{D}(A) \rightarrow \mathrm{CH}^{*}(A)_{\text {num }, \mathbf{Q}}
$$

factors through the Grothendieck group $K(A)$ of locally free sheaves via the Chern character

$$
\text { ch : } K(A) \rightarrow \mathrm{CH}^{*}(A)_{\text {num }, \mathbf{Q}} \text {. }
$$

Finally, we denote the Mukai pairing on $\mathrm{CH}^{*}(A)_{\text {num, }} \mathbf{Q}$ by

$$
\left\langle(r, l, \chi),\left(r^{\prime}, l^{\prime}, \chi^{\prime}\right)\right\rangle_{A}:=l \cdot l^{\prime}-r \chi^{\prime}-\chi r^{\prime},
$$

so that by the Grothendieck-Riemann-Roch Theorem there are equalities

$$
\langle v(\mathcal{F}), v(\mathcal{G})\rangle_{A}=-\chi(\mathcal{F}, \mathcal{G}) \text { for any objects } \mathcal{F}, \mathcal{G} \text { in } \mathbf{D}(A)
$$

(as usual $\chi(\mathcal{F}, \mathcal{G})=\sum_{i}(-1)^{i} \operatorname{dim} \operatorname{Hom}_{\mathbf{D}(A)}^{i}(\mathcal{F}, \mathcal{G})$ ).

Given another abelian surface $B$, a Fourier-Mukai functor $\Phi_{\mathcal{E}}: \mathbf{D}(A) \rightarrow \mathbf{D}(B)$ induces a group homomorphism $\Phi_{\mathcal{E}}^{\mathrm{CH}}: \mathrm{CH}^{*}(A)_{\text {num, }, \mathbf{Q}} \rightarrow \mathrm{CH}^{*}(B)_{\text {num, }} \mathbf{Q}$ through the formula

$$
\Phi_{\mathcal{E}}^{\mathrm{CH}}(-):=\operatorname{pr}_{2 *}\left(\operatorname{pr}_{1}^{*}(-) \cdot v(\mathcal{E})\right)
$$

where $\mathrm{pr}_{1}$ and $\mathrm{pr}_{2}$ denote the projections from the product $A \times B$ onto the first and second factor respectively. We point out that in general $\Phi_{\mathcal{E}}^{\mathrm{CH}}$ does not respect the grading. As showed 
in [7] and [10, Sect. 3], we have that $\left(\Phi_{\mathcal{E}} \circ \Phi_{\mathcal{E}^{\prime}}\right)^{\mathrm{CH}} \simeq \Phi_{\mathcal{E}}^{\mathrm{CH}} \circ \Phi_{\mathcal{E}^{\prime}}^{\mathrm{CH}}$, and that $\Phi_{\mathcal{E}}^{\mathrm{CH}}$ is invertible if $\Phi_{\mathcal{E}}$ is an equivalence. Finally, we note that if $\Phi_{\mathcal{E}}$ is an equivalence, then it follows that

$$
\left\langle\Phi_{\mathcal{E}}^{\mathrm{CH}}(v(\mathcal{F})), \Phi_{\mathcal{E}}^{\mathrm{CH}}(v(\mathcal{G}))\right\rangle_{B}=\langle v(\mathcal{F}), \quad v(\mathcal{G})\rangle_{A}
$$

from (2.1), and by the fact that $v \circ \Phi_{\mathcal{E}}=\Phi_{\mathcal{E}}^{\mathrm{CH}} \circ v$.

We conclude this subsection by pointing out the following peculiar fact true for abelian surfaces. Its proof is identical to that of [7, Corollary 9.43] with the opportune modifications. Moreover it holds in any dimension.

Proposition 2.1 If $\Phi: \mathbf{D}(A) \rightarrow \mathbf{D}(B)$ is an equivalence of derived categories of abelian surfaces, then

$$
\Phi^{\mathrm{CH}}\left(\mathrm{CH}^{*}(A)_{\text {num }}\right)=\mathrm{CH}^{*}(B)_{\text {num }} .
$$

We will tacitly use the previous result throughout the rest of the paper.

Notation 2.2 Given an abelian surface $A$ over $k$ we denote by $\mathrm{CH}^{*}(A)=\oplus_{i} \mathrm{CH}^{i}(A)$ the graded ring $\mathrm{CH}^{*}(A)_{\text {num }}$.

\subsection{Some examples of (auto)equivalences}

We denote by $A$ an abelian surface and by $\widehat{A}$ its dual variety. Moreover let $\mathcal{P}$ be the normalized Poincaré line bundle on $A \times \widehat{A}$ so that $\mathcal{S}_{A}:=\Phi_{\mathcal{P}}: \mathbf{D}(A) \rightarrow \mathbf{D}(\widehat{A})$ is an equivalence of triangulated categories [23]. The action of $\mathcal{S}_{A}^{\mathrm{CH}}$ swaps the first and third entry of a vector, e.g.:

$\mathcal{S}_{A}^{\mathrm{CH}}\left(\mathrm{CH}^{0}(A)\right)=\mathrm{CH}^{2}(\widehat{A}), \quad \mathcal{S}_{A}^{\mathrm{CH}}\left(\mathrm{CH}^{2}(A)\right)=\mathrm{CH}^{0}(\widehat{A}), \quad$ and $\quad \mathcal{S}_{A}^{\mathrm{CH}}\left(\mathrm{CH}^{1}(A)\right)=\mathrm{CH}^{1}(\widehat{A})$.

Let now $H$ be a line bundle on $A$ and $h$ be its class in $\mathrm{CH}^{1}(A)$. The autoequivalence $T_{A}\left(H^{\otimes n}\right)$ : $\mathbf{D}(A) \rightarrow \mathbf{D}(A)(n \in \mathbf{Z})$ defined by $\mathcal{F} \mapsto \mathcal{F} \otimes H^{\otimes n}$ acts on the numerical Chow rings as:

$$
T_{A}\left(H^{\otimes n}\right)^{\mathrm{CH}}(r, l, \chi)=\left(r, l+r n h, \chi+n l \cdot h+r n^{2} \frac{h^{2}}{2}\right) .
$$

Finally, the shift functor [1] $: \mathbf{D}(A) \rightarrow \mathbf{D}(A)$ acts on $\mathrm{CH}^{*}(A)$ by -1 .

\subsection{Isogenies and exponents}

If $A$ is an abelian variety over $k$, we denote by $n_{A}: A \rightarrow A$ the multiplication-by- $n$-map and by $A[n]$ its kernel. We say that an elliptic curve $E$ over $k$ is ordinary (resp. supersingular) if $E[p](k)=\mathbf{Z} / p \mathbf{Z}$ (resp. $E[p](k)=0)$ (cf. [16]). Therefore $E$ is supersingular if and only if $p_{E}$ is inseparable and the $j$-invariant is defined over $\mathbf{F}_{p^{2}}$, the finite field with $p^{2}$ elements (cf. [33, Theorem V.3.1]). The exponent $\exp \varphi$ of a separable isogeny $\varphi: A \rightarrow B$ of abelian varieties is the smallest positive integer that annihilates its kernel. Finally we recall that if $\varphi: A \rightarrow B$ is an isogeny of exponent $e$, then there exists an isogeny $\psi: B \rightarrow A$ of exponent $e$ such that $\psi \circ \varphi=e_{A}$ and $\varphi \circ \psi=e_{B}$ (cf. [3, Proposition 1.2.6]).

Proposition 2.3 Let $v: A \rightarrow B$ be a separable isogeny of exponent $e$ and denote by $v^{*}$ : $\mathrm{CH}^{1}(B) \rightarrow \mathrm{CH}^{1}(A)$ the pull-back homomorphism. Then there is an inclusion of groups $e^{2} \mathrm{CH}^{1}(A) \subset \operatorname{Im}\left(v^{*}\right)$. 
Proof Let $\mu: B \rightarrow A$ be the isogeny such that $\mu \circ v=e_{A}$ and note that $\operatorname{Im}\left(e_{A}^{*}: \mathrm{CH}^{1}(A) \rightarrow\right.$ $\left.\mathrm{CH}^{1}(A)\right) \subset \operatorname{Im}\left(v^{*}\right)$. We conclude by using [21, Remark 10.19] which shows that for any line bundle $L, e_{A}^{*} L$ is algebraically equivalent, hence numerically equivalent, to $L^{\otimes e^{2}}$.

Proposition 2.4 If $E$ and $F$ are supersingular elliptic curves and $l \neq p=\operatorname{char}(k)$ is a prime, then there exists an integer $r \gg 0$ and a separable isogeny $\xi: F \rightarrow E$ of degree $l^{r}$.

Proof Since $E$ and $F$ are supersingular, their $j$-invariants are defined over $\mathbf{F}_{p^{2}}$. Moreover by [14, Corollary 78] there exists an isogeny $\xi^{\prime}: F_{\mathbf{F}_{p^{2}}} \rightarrow E_{\mathbf{F}_{p^{2}}}$ of degree $l^{r}$ for some positive integer $r \gg 0$. Therefore we obtain our desired isogeny from $\xi^{\prime}$ by extension of scalars. Finally we observe that $\xi$ is separable as the degree of every non-separable isogeny is divisible by char $(k)$ [33, Corollary 2.12].

\subsection{Line bundles on a product of two elliptic curves}

Let $\left(E, O_{E}\right)$ be an elliptic curve over $k$. We denote the Mumford bundle on $E \times E$ by

$$
\mathcal{M}_{E}=\mathcal{O}_{E \times E}\left(\Delta_{E}\right) \otimes \operatorname{pr}_{1}^{*} \mathcal{O}_{E}\left(-O_{E}\right) \otimes \operatorname{pr}_{2}^{*} \mathcal{O}_{E}\left(-O_{E}\right)
$$

where $\Delta_{E} \subset E \times E$ is the diagonal divisor and $\mathrm{pr}_{1}, \mathrm{pr}_{2}$ are the projections of $E \times E$ onto the first and second factor respectively. Given another elliptic curve $\left(F, O_{F}\right)$, line bundles $L_{E}$ and $L_{F}$ on $E$ and $F$ respectively, and a morphism $\varphi: F \rightarrow E$, we define a line bundle on the product $E \times F$

$$
L\left(\varphi, L_{E}, L_{F}\right):=\left(1_{E} \times \varphi\right)^{*} \mathcal{M}_{E} \otimes \operatorname{pr}_{E}^{*} L_{E} \otimes \operatorname{pr}_{F}^{*} L_{F}
$$

where $\mathrm{pr}_{E}$ and $\mathrm{pr}_{F}$ are the projections onto $E$ and $F$ respectively.

Proposition 2.5 If $\varphi: F \rightarrow E$ and $\psi: F \rightarrow E$ are isogenies, then

$$
\left(1_{E} \times(\varphi+\psi)\right)^{*} \mathcal{M}_{E} \simeq\left(1_{E} \times \varphi\right)^{*} \mathcal{M}_{E} \otimes\left(1_{E} \times \psi\right)^{*} \mathcal{M}_{E} .
$$

Therefore for any choice of line bundles $M_{E}$ and $N_{E}$ on $E$, and line bundles $M_{F}$ and $N_{F}$ on $F$, there are isomorphisms

$$
L\left(\varphi+\psi, M_{E} \otimes N_{E}, M_{F} \otimes N_{F}\right) \simeq L\left(\varphi, M_{E}, M_{F}\right) \otimes L\left(\psi, N_{E}, N_{F}\right) .
$$

Proof The proof is a simple application of the see-saw principle.

If $L_{E}, L_{E}^{\prime}$ and $L_{F}, L_{F}^{\prime}$ are line bundles on $E$ and $F$ respectively such that $d_{E}:=\operatorname{deg} L_{E}=$ $\operatorname{deg} L_{E}^{\prime}$ and $d_{F}:=\operatorname{deg} L_{F}=\operatorname{deg} L_{F}^{\prime}$, then the classes of $L\left(\varphi, L_{E}, L_{F}\right)$ and $L\left(\varphi, L_{E}^{\prime}, L_{F}^{\prime}\right)$ coincide in $\mathrm{CH}^{1}(E \times F)$. We denote by $l\left(\varphi, d_{E}, d_{F}\right)$ the numerical class of $L\left(\varphi, L_{E}, L_{F}\right)$ (or $L\left(\varphi, L_{E}^{\prime}, L_{F}^{\prime}\right)$ ).

Corollary 2.6 With notation as in Proposition 2.5, in $\mathrm{CH}^{1}(E \times F)$ there are equalities of classes

$$
l\left(\varphi+\psi, d_{E}+d_{E}^{\prime}, d_{F}+d_{F}^{\prime}\right)=l\left(\varphi, d_{E}, d_{F}\right)+l\left(\psi, d_{E}^{\prime}, d_{F}^{\prime}\right)
$$

where $d_{E}$ and $d_{F}$ are the degrees of $M_{E}$ and $M_{F}$ respectively, and $d_{E}^{\prime}$ and $d_{F}^{\prime}$ are the degrees of $N_{E}$ and $N_{F}$ respectively.

Finally we show that any line bundle $L \in \operatorname{Pic}(E \times F)$ can be realized as a line bundle of the form (2.4). 
Proposition 2.7 For any line bundle $L \in \operatorname{Pic}(E \times F)$ there exists a morphism $\varphi: F \rightarrow E$ and line bundles $L_{E} \in \operatorname{Pic}(E)$ and $L_{F} \in \operatorname{Pic}(F)$ such that $L \simeq L\left(\varphi, L_{E}, L_{F}\right)$.

Proof Denote by $L_{E}$ the restriction of $L$ to $E \times\left\{O_{F}\right\}$, and similarly let $L_{F}$ be the restriction of $L$ to $\left\{O_{E}\right\} \times F$. Set now $L^{\prime}:=L \otimes \operatorname{pr}_{E}^{*} L_{E}^{-1} \otimes \operatorname{pr}_{F}^{*} L_{F}^{-1}$. We note that the restriction of $L^{\prime}$ to $\left\{O_{E}\right\} \times F$ is trivial, while the restrictions $\left.L^{\prime}\right|_{E \times\{y\}}$ lie in $\operatorname{Pic}^{0}(E)$ for all $y \in F$. Thus by the universal property of the dual variety [26, Theorem on p. 117], there exists a unique morphism $\tilde{\varphi}: F \rightarrow \widehat{E}$ such that

$$
L^{\prime}=\left(1_{E} \times \widetilde{\varphi}\right)^{*} \mathcal{P}_{E}
$$

where $\mathcal{P}_{E}$ is the normalized Poincare line bundle on $E \times \widehat{E}$ (namely the restrictions of $\mathcal{P}_{E}$ to $\left\{O_{E}\right\} \times \widehat{E}$ and $E \times\left\{O_{\widehat{E}}\right\}$ are trivial). Moreover we have an isomorphism $\mathcal{M}_{E} \simeq\left(1_{E} \times \eta\right)^{*} \mathcal{P}_{E}$ induced by the isomorphism $\eta(x)=\mathcal{O}_{E}\left(x-O_{E}\right)$. Hence $L^{\prime} \simeq\left(1_{E} \times\left(\eta^{-1} \widetilde{\varphi}\right)\right)^{*} \mathcal{M}_{E}$ and the conclusion of the proposition follows by setting $\varphi=\eta^{-1} \widetilde{\varphi}$.

\subsection{Lifting results}

Let $k$ be a perfect field of positive characteristic $p$ and let $W(k)$ be the ring of Witt vectors with quotient field $K$. We recall that it is a complete discrete valuation ring such that $K$ is of characteristic zero (see for instance [31, Sect. II.6]). We will denote by $W$ both $W(k)$ and finite extensions of $W(k)$. If $X$ denotes a smooth projective scheme over $k$, a projective lift of $X$ over $W$ is a flat morphism $\psi: \mathcal{X} \rightarrow W$ where $\mathcal{X}$ is a projective scheme, together with the choice of an isomorphism between the closed fiber $\mathcal{X}_{k}$ and $X$. Grothendieck's existence theorem establishes that smooth curves always lift, as well as the line bundles on them. Moreover ordinary abelian varieties admit a canonical lift over $W$ characterized by the fact that the absolute Frobenius lifts with the abelian variety (we recall that an abelian variety $A$ is ordinary if $\left.A[p](k) \simeq(\mathbf{Z} / p \mathbf{Z})^{\operatorname{dim} A}\right)$. We refer to [22, Appendix, Theorem 1] for the proof of the following result.

Theorem 2.8 Let A be an ordinary abelian variety over a perfect field $k$ of positive characteristic $p$. Then there exists a projective lift $\mathcal{A} \rightarrow W$ of A together with a morphism $F_{\mathcal{A}}: \mathcal{A} \rightarrow \mathcal{A}$ compatible with the Frobenius of $W$ such that $F_{\mathcal{A} \mid A}$ is the absolute Frobenius $F_{A}$ of $A$. The pair $\left(\mathcal{A}, F_{\mathcal{A}}\right)$ is called a canonical lift and is unique up to a unique isomorphism inducing the identity on $A$. Moreover, the restriction morphism $\operatorname{Pic}(\mathcal{A}) \rightarrow \operatorname{Pic}(A)$ is surjective and

$$
\operatorname{Pic}(\mathcal{A})_{F_{\mathcal{A}}}:=\left\{\mathcal{L} \in \operatorname{Pic}(\mathcal{A}) \mid F_{\mathcal{A}}^{*} \mathcal{L} \simeq \mathcal{L}^{\otimes p}\right\} \simeq \operatorname{Pic}(A) .
$$

Finally, if $\varphi: A \rightarrow B$ is a morphism between ordinary abelian varieties, then there exists a unique morphism $\tilde{\varphi}: \mathcal{A} \rightarrow \mathcal{B}$ of canonical liftings such that $F_{\mathcal{B}} \circ \tilde{\varphi}=\widetilde{\varphi} \circ F_{\mathcal{A}}$ and $\tilde{\varphi}_{\mid A}=\varphi$.

Another result that we will need in the sequel is the existence of liftings of étale covers. A reference for the following theorem is [32, Sect. IX, 1.10].

Theorem 2.9 Let $S$ be the spectrum of a complete local Noetherian ring, and let $X \rightarrow S$ be a proper $S$-scheme. Moreover denote by $X_{0}$ the closed fiber over the unique closed point of $S$. Then the assignment $X^{\prime} \mapsto X^{\prime} \times_{X} X_{0}$ yields an equivalence between the category of finite étale coverings of $X$ and the category of finite étale coverings of $X_{0}$. 


\subsection{Moduli spaces}

Let $A$ be an abelian surface defined over a field $k$ and let $h \in \mathrm{NS}(A)$ be the class of an ample line bundle. Given a vector $v=(r, l, \chi) \in \mathrm{CH}^{*}(A)$ with integral coefficients, we consider the moduli space $\mathcal{M}_{h}(v)$ of Gieseker-semistable sheaves with Mukai vector $v$, where stability is computed with respect to $h$. We want to show that, under certain assumptions, these spaces are irreducible and are Fourier-Mukai partners of $A$. We will need the following criterion for fully faithfulness:

Theorem 2.10 [19, Theorem 2.6] A Fourier-Mukai functor $\Phi_{\mathcal{U}}: \mathbf{D}(M) \rightarrow \mathbf{D}(A)$ between the derived categories of two smooth abelian surfaces over $k$ is fully faithful if the following three conditions are satisfied:

(i) $\operatorname{Hom}^{i}\left(\Phi_{\mathcal{U}}(k(x)), \Phi_{\mathcal{U}}(k(y))=0\right.$ if $0>i$ or $i>\operatorname{dim} X$ or when $x \neq y$;

(ii) $\operatorname{Hom}^{0}\left(\Phi_{\mathcal{U}}(k(x)), \Phi_{\mathcal{U}}(k(x))=k\right.$ for all $x \in M$;

(iii) $\Phi_{\mathcal{U}^{\vee}[2]} \circ \Phi_{\mathcal{U}}(k(x)) \simeq k(x)$ for some $x \in M$.

Now we can turn to the proof of the following:

Theorem 2.11 If $r>0$ and $\chi$ are coprime integers, then every Gieseker-semistable sheaf on $A$ with Mukai vector $v$ is Gieseker-stable. Moreover, if in addition $\langle v, v\rangle_{A}=0$, then $\mathcal{M}_{h}(v)$ is a smooth irreducible projective variety of dimension two which admits a universal family $\mathcal{U}$ on $\mathcal{M}_{h}(v) \times A$. Furthermore, $\mathcal{M}_{h}(v)$ has trivial canonical bundle and the Fourier-Mukai functor $\Phi_{\mathcal{U}}: \mathbf{D}\left(\mathcal{M}_{h}(v)\right) \rightarrow \mathbf{D}(A)$ induces an equivalence of derived categories.

Proof The first assertion follows by [8, Remark 4.6.8] (cf. also [8, Remark 6.1.9]), while the second follows by [24, Corollary 0.2]. The existence of a universal sheaf follows by [25, Theorem A.6]. We remark, as noted by Lieblich-Olsson [17, Remark 3.17], that while the results of [25] are just stated for characteristic 0, the argument is true also in positive characteristic. The only tricky point in the algebraic setting is determining the non-emptiness of these spaces, which can be done using the work of Langton [15, Theorem on p. 99].

To prove the irreducibility of $\mathcal{M}_{h}(v)$, we use an argument of Mukai. Let $M \subseteq \mathcal{M}_{h}(v)$ be an irreducible component. The smoothness of $\mathcal{M}_{h}(v)$ implies that $M$ is also a connected component of the moduli space. Suppose that $M \neq \mathcal{M}_{h}(v)$. Let $\mathcal{U}$ be the universal family of $\mathcal{M}_{h}(v)$ restricted to $M \times A$. We can construct the Fourier-Mukai functor

$$
\Phi_{\mathcal{U}^{\vee}[2]}: \mathbf{D}(A) \rightarrow \mathbf{D}(M) .
$$

Observe that we have an isomorphism of functors $\Phi_{\mathcal{U}^{\vee}[2]}(-) \simeq \mathbf{R} p_{1 *} \mathbf{R} \mathcal{H o m}\left(\mathcal{U}, p_{2}^{*}(-)\right)[2]$. This functor sends any stable sheaf on $A$ whose corresponding point lies on $M \subset \mathcal{M}_{h}(v)$ to a skyscraper sheaf at that point on $M$, while it sends all the stable sheaves on $A$ that are not on $M$ to zero (see for example [13, Proof of Theorem 2.2] - the proof uses only that there are no nontrivial homs between stable sheaves of the same slope so it works also in positive characteristic). On the other hand, for any sheaf $\mathcal{F}$ on $M$, the Mukai vector of $\Psi_{\mathcal{U}}(\mathcal{F})$ only depends on the Mukai vector of $\mathcal{F}$ as it is obtained by convoluting $v(\mathcal{F})$ with $v\left(\mathcal{U}^{\vee}\right)$. This leads to a contradiction as skyscraper sheaves and the zero object have different Mukai vectors.

Finally, we only have to show that $\Phi_{\mathcal{U}}$ is an equivalence. We will prove that it is fully faithful using Theorem 2.10, and then conclude by [7, Proposition 7.6]. We remark that, even though Chapter 7 of [7] is in characteristic 0 , this proposition uses only the fully-faithfulness of $\Phi_{\mathcal{U}}$ and some general results about $k$-linear categories and adjoint functors, so it works in our setting. 
By [25, Proposition 3.12] the universal family $\mathcal{U}$ is strongly simple. In particular we have that

(i) $\operatorname{Hom}^{i}\left(\Phi_{\mathcal{U}}(k(x)), \Phi_{\mathcal{U}}(k(y))=0\right.$ if $0>i$ or $i>\operatorname{dim} X$ or when $x \neq y$;

(ii) $\operatorname{Hom}^{0}\left(\Phi_{\mathcal{U}}(k(x)), \Phi_{\mathcal{U}}(k(x))=k\right.$.

So we just need to check that there is a skyscraper sheaf such that

$$
\Phi_{\mathcal{U}^{\vee}[2]} \circ \Phi_{\mathcal{U}}(k(x)) \simeq k(x) .
$$

By the definition of universal family the functor $\Phi_{\mathcal{U}}$ sends a skyscraper sheaf $k(x)$ to a stable sheaf $\mathcal{U}_{x} \in \mathcal{M}_{h}(v)$. The above discussion implies that $\mathcal{E}:=\Phi_{\mathcal{U}^{\vee}[2]}\left(\mathcal{U}_{x}\right)$ is a skyscraper sheaf on $\mathcal{M}_{h}(v)$. Since $\Phi_{\mathcal{U}^{\vee}[2]}$ is the left adjoint of $\Phi_{\mathcal{U}}$, the strong simplicity of $\mathcal{U}$ implies that $\operatorname{Hom}_{\mathbf{D}\left(\mathcal{M}_{h}(v)\right)}(\mathcal{E}, k(x)) \simeq k$, and so we are finished.

\subsection{Relative moduli spaces}

We also need to consider relative moduli spaces of Gieseker-semistable sheaves on a projective lift $f: \mathcal{A} \rightarrow W$ of an abelian surface $A$ over the ring of Witt vectors. Let $h$ be the class of an ample line bundle as before, and let $\widetilde{h}$ be a lifting of $h$ to $\mathcal{A}$. Let $v=(r, l, \chi) \in \mathrm{CH}^{*}(A)$ be a vector with integral coefficients such that $l$ is the class of a line bundle $L$ that lifts to a line bundle $\widetilde{L}$ on $\mathcal{A}$. Moreover set $\widetilde{v}=(r, \widetilde{l}, \chi)$ where $\widetilde{l}$ is the class of $\widetilde{L}$. By [20, Theorem 0.7] (or [15, Theorem 0.2]) there exists a projective scheme $\mathcal{M}_{\mathcal{A} / W}(\widetilde{v}) \rightarrow W$ of finite type that is a coarse moduli space for the functor of families of pure Gieseker-semistable sheaves with Mukai vector $v$ on the geometric fibers of $f$ (where stability is computed with respect to $\widetilde{h}$ ). Moreover, there exists an open subscheme $\mathcal{M}_{\mathcal{A} / W}^{s}(\widetilde{v}) \subset \mathcal{M}_{\mathcal{A} / W}(\widetilde{v})$ that is a coarse moduli space for the subfunctor of families of pure Gieseker-stable sheaves. Thus, if $(r, \chi)=1$ (i.e. every Gieseker-semistable sheaf is Gieseker-stable), then we have $\mathcal{M}_{\mathcal{A} / W}^{s}(\widetilde{v})=\mathcal{M}_{\mathcal{A} / W}(\widetilde{v})$. Note that if we denote the closed fiber of $f$ by $A_{k}$ and the geometric generic fiberby $A_{\eta}$, then there are isomorphisms

$$
\mathcal{M}_{\mathcal{A} / W}(\widetilde{v})_{\mid A_{k}} \simeq \mathcal{M}_{h}(v) \text { and } \mathcal{M}_{\mathcal{A} / W}(\widetilde{v})_{\mid A_{\eta}} \simeq \mathcal{M}_{h_{\eta}}\left(v_{\eta}\right)
$$

where $\mathcal{M}_{h_{\eta}}\left(v_{\eta}\right)$ is the moduli space of pure Gieseker-stable sheaves with vector $v_{\eta}=$ $\left(r, \tilde{l}_{\mid A_{\eta}}, \chi\right)$ on $A_{\eta}$, and $h_{\eta}$ is the restriction of $\widetilde{h}$ to $A_{\eta}$. The smoothness of the moduli space implies that $\tilde{f}: \mathcal{M}_{\mathcal{A} / W}(\widetilde{v}) \rightarrow W$ is formally smooth and of finite type, and hence flat. So, with a choice of isomorphism $\mathcal{M}_{\mathcal{A} / W}(\widetilde{v})_{\mid A_{k}} \simeq \mathcal{M}_{h}(v)$, it is a lift of $\mathcal{M}_{h}(v)$.

\section{Filtered derived equivalences}

An equivalence $\Phi: \mathbf{D}(A) \rightarrow \mathbf{D}(B)$ of derived categories of abelian surfaces is filtered if

$$
\Phi^{\mathrm{CH}}(0,0,1)=(0,0,1) \text {. }
$$

In [17, Theorem 6.1] the authors prove that if two $K 3$ surfaces have a filtered equivalence between them, then they are isomorphic. The proof of this statement is quite involved and uses deformation theory of complexes in order to lift a derived equivalence of $K 3$ surfaces in positive characteristic to an equivalence of $K 3$ surfaces in characteristic zero. Here we notice that a filtered equivalence of abelian surfaces still induces an isomorphism. As the kernel of an equivalence of abelian varieties is a sheaf (up to shift), its proof turns out to be rather simple. 
Proposition 3.1 Let $\Phi: \mathbf{D}(A) \rightarrow \mathbf{D}(B)$ be a filtered equivalence between the derived categories of two abelian surfaces. Then there exists an isomorphism $f: A \rightarrow B$ and a line bundle $L$ on $A \times B$ such that $\Phi$ is isomorphic to $(-\otimes L) \circ f_{*}$ up to shift. In particular $A$ and $B$ are isomorphic.

Proof Equivalences of derived categories of abelian varieties send (up to shift) structure sheaves of points $\mathcal{O}_{x}$ to sheaves. This is proved in [5, Lemma 10.2.6] in characteristic zero, but its proof extends to positive characteristic without any change. Hence we can suppose that $\Phi\left(\mathcal{O}_{x}\right)$ is a sheaf with Mukai vector $(0,0,1)$, so it is itself a skyscraper sheaf. Since the argument holds for all points $x$ in $A$, the proposition follows by [7, Corollary 5.23].

We now prove Theorem 1.2 of the introduction, which relies on the following technical proposition.

Proposition 3.2 Let $\Phi: \mathbf{D}(A) \rightarrow \mathbf{D}(B)$ be an equivalence between the derived categories of two abelian surfaces. Then there exists an equivalence $\Psi: \mathbf{D}(A) \rightarrow \mathbf{D}(C)$ where $C \in\{B, \widehat{B}\}$ such that the vector

$$
\Psi^{\mathrm{CH}}(0,0,1):=(r, l, \chi)
$$

satisfies the following conditions:

(i) $r$ is positive;

(ii) $l$ is the class of an ample line bundle on $C$;

(iii) $r$ is coprime with $\chi$.

Moreover we have $C=B$ (resp. $C=\widehat{B}$ ) if the first component of $\Phi^{\mathrm{CH}}(0,0,1)$ is nonzero (resp. zero).

\section{Proof Set}

$$
v_{0}:=\Phi^{\mathrm{CH}}(0,0,1)=\left(r_{0}, l_{0}, \chi_{0}\right) .
$$

We split our argument in several steps.

Step 1 Given an equivalence $\Phi: \mathbf{D}(A) \rightarrow \mathbf{D}(B)$ as in the statement, there exists an equivalence $\Psi_{1}: \mathbf{D}(A) \rightarrow \mathbf{D}(C)$ with $C \in\{B, \widehat{B}\}$ such that the first entry of the vector

$$
v_{1}:=\Psi_{1}^{\mathrm{CH}}(0,0,1)=\left(r_{1}, l_{1}, \chi_{1}\right)
$$

is positive. Moreover we have $C=B$ if $r_{0} \neq 0$, and $C=\widehat{B}$ otherwise.

If $r_{0}>0$ there is nothing to prove and we simply set $\Psi_{1}=\Phi: \mathbf{D}(A) \rightarrow \mathbf{D}(B)$. If $r_{0}<0$, we set $\Psi_{1}:=\Phi \circ[1]: \mathbf{D}(A) \rightarrow \mathbf{D}(B)$ in order to make $r_{0}$ positive. Suppose now that $r_{0}=0$ and $\chi_{0} \neq 0$. Then it is enough to set $\Psi_{1}:=\mathcal{S}_{B} \circ \Phi$ if $\chi_{0}>0$, and $\Psi_{1}:=\mathcal{S}_{B} \circ \Phi[1]$ if $\chi_{0}<0$. Observe that both these equivalences are between $\mathbf{D}(A)$ and $\mathbf{D}(\widehat{B})$. So we are left with the case $r_{0}=\chi_{0}=0$. Let $\Phi^{\mathrm{CH}}(1,0,0):=w_{0}=\left(s_{0}, b_{0}, \xi_{0}\right)$. By noting that $v\left(\mathcal{O}_{x}\right)=(0,0,1)$ for any point $x \in B$, and $v\left(\mathcal{O}_{B}\right)=(1,0,0)$, by $(2.2)$ we find that $\left\langle v_{0}, w_{0}\right\rangle_{B}=l_{0} \cdot b_{0}=1$. Hence, if $B_{0}$ is a line bundle with class $b_{0}$, then the composition $\left(T_{B}\left(B_{0}\right) \circ \Phi\right)^{\mathrm{CH}}$ sends $(0,0,1)$ to $\left(0, l_{0}, 1\right)$ as showed in (2.3). Thus we set $\Psi_{1}:=\mathcal{S}_{B} \circ T_{B}\left(B_{0}\right) \circ \Phi: \mathbf{D}(A) \rightarrow \mathbf{D}(\widehat{B})$ and we have the desired equivalence.

Step 2 Given an equivalence $\Psi_{1}: \mathbf{D}(A) \rightarrow \mathbf{D}(C)$ as in the previous step, there exists an equivalence $\Psi_{2}: \mathbf{D}(A) \rightarrow \mathbf{D}(C)$ so that in $v_{2}:=\Psi_{2}^{\mathrm{CH}}(0,0,1)=\left(r_{2}, l_{2}, \chi_{2}\right), r_{2}>0$ and $\chi_{2}$ is coprime with $r_{2}$. 
Let $w_{1}:=\Psi_{1}^{\mathrm{CH}}(1,0,0)=\left(s_{1}, b_{1}, \xi_{1}\right)$, so $\left\langle v_{1}, w_{1}\right\rangle_{C}=1$ and

$$
I-r_{1} \xi_{1}-\chi_{1} s_{1}=1 \text { where } \quad I:=l_{1} \cdot b_{1} .
$$

By (3.1), it is possible to choose an $n$ so that $\chi_{1}+n I$ is relatively prime with $r_{1}$. Let $B_{1}$ be a line bundle with numerical class $b_{1}$. By (2.3) the homomorphism $\left(T_{C}\left(B_{1}^{\otimes n}\right) \circ \Psi_{1}\right)^{\mathrm{CH}}$ sends $(0,0,1)$ to

$$
v_{2}:=\left(r_{2}, l_{2}, \chi_{2}\right)=\left(r_{1}, l_{1}+r_{1} n b_{1}, \chi_{1}+n I+r_{1} n^{2} \frac{b_{1}^{2}}{2}\right) .
$$

Our choice of $n$ ensures that $r_{2}$ and $\chi_{2}$ are relatively prime.

Step 3 Given an equivalence $\Psi_{2}: \mathbf{D}(A) \rightarrow \mathbf{D}(C)$ as in the previous step, there exists an equivalence $\Psi_{3}: \mathbf{D}(A) \rightarrow \mathbf{D}(C)$ satisfying the conditions of the proposition.

Let $\Theta$ be an ample line bundle with class $\theta \in \mathrm{CH}^{1}(C)$. For any integer $d>0$, the equivalence $T_{C}\left(\Theta^{\otimes\left(r_{1} d\right)}\right) \circ \Psi_{2}$ sends $(0,0,1)$ to

$$
\left(r_{1}, l_{1}+r_{1} n b_{1}+r_{1}^{2} d \theta, \chi_{1}+n I+r_{1} n^{2} \frac{b_{1}^{2}}{2}+r_{1} d\left(\theta \cdot\left(l_{1}+r_{1} n b_{1}\right)\right)+r_{1}^{3} d^{2} \frac{\theta^{2}}{2}\right) \text {. }
$$

Choose $d$ large enough so that the second component is an ample class and set $\Psi_{3}^{\mathrm{CH}}:=$ $T_{C}\left(\Theta^{\otimes\left(r_{1} d\right)}\right) \circ \Psi_{2}$. The first component of $v_{3}:=\Psi_{3}^{\mathrm{CH}}(0,0,1)$ is positive by the previous steps, and the third component of $v_{3}$ is congruent to $\chi_{2}$ modulo $r_{1}$.

Theorem 3.3 Let $A$ be an abelian surface and let $\Phi: \mathbf{D}(A) \rightarrow \mathbf{D}(Y)$ be an equivalence of derived categories of smooth projective varieties. Then $Y$ is an abelian surface. Moreover, $A$ is isomorphic to a moduli space of Gieseker-stable sheaves on either $Y$, or its dual $\widehat{Y}$, according to whether the rank component of $\Phi^{\mathrm{CH}}(0,0,1)$ is non-zero, or zero, respectively.

Proof By general theory $Y$ has trivial canonical bundle and Kodaira dimension 0 . Let $l \neq p$ be a prime and consider the $\ell$-adic cohomology groups $H_{e}^{i}\left(Y, \mathbf{Q}_{\ell}\right)$. By [9, Lemma 3.1] the equivalence $\Phi$ induces an isomorphism

$$
H_{\grave{e} t}^{1}\left(Y, \mathbf{Q}_{\ell}\right) \oplus H_{e}^{3}\left(Y, \mathbf{Q}_{\ell}\right)(2) \simeq H_{e}^{1}\left(A, \mathbf{Q}_{\ell}\right) \oplus H_{e}^{3}\left(A, \mathbf{Q}_{\ell}\right)(2)
$$

which leads to the equality of Betti numbers:

$$
b_{1}(Y)=b_{1}(A)=4 .
$$

Since $Y$ is a smooth, projective surface with Kodaira dimension 0 and $b_{1}=4$, it is isomorphic to an abelian surface (See Theorem 6 and the table on p. 25 of [4]).

By Proposition 3.2 we may fix an equivalence $\Psi: \mathbf{D}(A) \rightarrow \mathbf{D}(C)$ with $C \in\{Y, \widehat{Y}\}$ such that in the vector

$$
v:=\Psi^{\mathrm{CH}}(0,0,1)=(r, l, \chi),
$$

the rank component $r$ is positive, the class $l$ is ample, and $\chi$ is coprime with $r$. Moreover, $C=Y$ if the rank component of $\Phi^{\mathrm{CH}}(0,0,1)$ is non-zero, and $C=\widehat{Y}$ otherwise.

By Theorem 2.11 there exists a universal family $\mathcal{U}$ on $\mathcal{M}_{l}(v) \times C$, which induces an equivalence

$$
\Phi_{\mathcal{U}}: \mathbf{D}\left(\mathcal{M}_{l}(v)\right) \rightarrow \mathbf{D}(C)
$$

such that $\Phi_{\mathcal{U}}^{\mathrm{CH}}(0,0,1)=v$. As the composition $\left(\Phi_{\mathcal{U}}^{-1} \circ \Psi\right)^{\mathrm{CH}}$ sends $(0,0,1)$ to $(0,0,1)$, by Proposition 3.1 we get $A \simeq \mathcal{M}_{l}\left(v_{l}\right)$. 


\section{FM partners of canonical covers of hyperelliptic surfaces}

In this and the next section, we will work, unless otherwise specified, under the assumption that the characteristic $p$ of the base field is bigger than 3 .

We denote the set of Fourier-Mukai partners of a smooth projective variety $X$ by

$$
\mathrm{FM}(X):=\{Y \mid Y \text { is a smooth projective variety with } \mathbf{D}(Y) \simeq \mathbf{D}(X)\}_{/ \simeq .}
$$

In the case of an abelian variety $A$, we say that its set of Fourier-Mukai partners is trivial if $\mathrm{FM}(A)=\{A, \widehat{A}\}$.

A hyperelliptic surface over an algebraically closed field $k$ of positive characteristic $p>3$ is a smooth projective minimal surface $X$ with $K_{X} \equiv 0, b_{2}(X)=2$, and such that each fiber of the Albanese map is a smooth elliptic curve (cf. [1, Sect. 10]). These surfaces can be described as quotients $(E \times F) / G$ of two elliptic curves $E$ and $F$ by a finite group $G$. The group $G$ acts on $E$ by translations, and on $F$ in a way such that $F / G \simeq \mathbf{P}^{1}$. Moreover, there are only a finite number of possibilities for the action of $G$ on $E \times F$, which have been classified by Bagnera-De Franchis [1, 10.27].

By [1, Sect. 9.3] the order $n$ of the canonical bundle of $X$ is finite with $n=2,3,4,6$. Therefore we can consider the canonical cover $\pi: \widetilde{X} \rightarrow X$ of the surface $X$, which is the étale cyclic cover associated to the canonical bundle $\omega_{X}$. The degree of $\pi$ is the order $n$ of $\omega_{X}$, and in addition $\pi$ comes equipped with an action of the cyclic group that realizes $X$ as the quotient $\widetilde{X} /(\mathbf{Z} / n \mathbf{Z})$. According to Bagnera-De Franchis' list [1, 10.27], the canonical cover $\widetilde{X}$ of an arbitrary hyperelliptic surface $X=(E \times F) / G$ is an abelian surface that sits inside a tower of surfaces

$$
E \times F \stackrel{\pi^{\prime}}{\longrightarrow} \tilde{X} \stackrel{\pi}{\longrightarrow} X,
$$

where $\pi^{\prime}$ is an étale cyclic cover of degree at most three. Moreover, if $\pi^{\prime}$ has degree three, then $F$ admits an automorphism group of order three and has $j$-invariant equals to zero. Therefore the dual morphism $\widehat{\pi^{\prime}}$ realizes the dual of $\widetilde{X}$ as one of the following varieties: $(i)$ the product $E \times F$, (ii) an étale cyclic cover of $E \times F$ of degree two, or ( $i i i)$ an étale cyclic cover of $E \times F$ of degree three such that $F$ has an automorphism group of order three.

\subsection{The work of Sosna}

In [34, Theorem 1.1] the author proves that the set of Fourier-Mukai partners of the canonical cover of a complex hyperelliptic surface is trivial. By using Bagnera-De Franchis' classification, Sosna's theorem boils down to proving the following result concerning derived equivalences of special abelian surfaces.

Theorem 4.1 (Sosna) Let $E$ and $F$ be complex elliptic curves and let $A$ be a complex abelian surface. Then the set $\mathrm{FM}(E \times F)$ is trivial. Moreover, if $E \times F \rightarrow A$ is a degree two étale cyclic cover, then $\operatorname{FM}(A)$ is trivial. Finally, the same conclusion holds if $E \times F \rightarrow A$ is a degree three étale cyclic cover and $\operatorname{rk} \operatorname{NS}(A) \in\{2,4\}$.

In view of Theorem 2.9 we prefer to work with étale covers rather than quotients. Thus we reformulate Sosna's theorem in the following version.

Proposition 4.2 Given a complex abelian surface A, then the set $\operatorname{FM}(A)$ is trivial in the following cases: 
(i) A is isomorphic to the product $E \times F$ of two elliptic curves;

(ii) $A$ is a degree two étale cyclic cover of a product $E \times F$ of two elliptic curves;

(iii) $A$ is a degree three étale cyclic cover of a product $E \times F$ of two elliptic curves and $\operatorname{rk} \operatorname{NS}(A) \in\{2,4\}$.

Proof If $A \rightarrow E \times F$ is a cover of degree one, two, or three, then the dual isogeny $E \times F \rightarrow \widehat{A}$ realizes $\widehat{A}$ as a quotient of two elliptic curves. Then by Theorem 4.1 we conclude that $\operatorname{FM}(\widehat{A})$ is trivial. $\operatorname{As} \operatorname{FM}(A)=\operatorname{FM}(\widehat{A})$ and $\operatorname{rk} \operatorname{NS}(A)=\operatorname{rkNS}(\widehat{A})$, the proposition follows at once.

As an application of Proposition 4.2, we deduce some further finitiness results that will be useful towards the proof of Theorem 1.1.

Proposition 4.3 If $\varphi: A \rightarrow E \times F$ is an isogeny with $\operatorname{deg} \varphi=8$ and $\exp \varphi=2$, then $\operatorname{FM}(A)$ is trivial. The same conclusion holds if $\operatorname{rk} \operatorname{NS}(A) \in\{2,4\}, \operatorname{deg} \varphi=27$ and $\exp \varphi=3$.

Proof We show that the dual abelian variety $\widehat{A}$ satisfies the hypotheses of Proposition 4.2. The result will follow as $\operatorname{FM}(A)=\operatorname{FM}(\widehat{A})$. Let $q$ be either 2 or 3 and consider an isogeny $\psi: E \times F \rightarrow A$ of exponent $q$ such that $\psi \circ \varphi=q_{A}$ (recall that $q_{A}$ denotes the multiplicationby- $q$-map on $A$ ). As $\operatorname{deg} q_{A}=q^{4}$ and $\operatorname{deg} \varphi=q^{3}$, we deduce that $\operatorname{deg} \psi=q$. Hence the dual isogeny $\widehat{\psi}$ is a cyclic cover of $E \times F$ of order $q$. The second statement follows as $\operatorname{rkNS}(A)=\operatorname{rkNS}(\widehat{A})$.

\subsection{Strategy of the proof of Theorem 1.1}

Since an abelian surface and its dual have the same Fourier-Mukai partners, the following theorem implies Theorem 1.1.

Theorem 4.4 Let A be an abelian surface over an algebraically closed field of characteristic $p>0$. Then $\mathrm{FM}(A)$ is trivial in the following cases:

(i) A is isomorphic to the product $E \times F$ of two elliptic curves;

(ii) $p>2$ and $A$ is a degree two étale cyclic cover over the product $E \times F$ of two elliptic curves;

(iii) $p>3$ and $A$ is a degree three étale cyclic cover over the product $E \times F$ of two elliptic curves such that $F$ admits an automorphism of order 3.

In order to prove the previous theorem, we will consider the following set of hypotheses

Setting 4.5 We denote by $E$ and $F$ two elliptic curves over an algebraically closed field $k$ of characteristic $p>0$. Moreover we set $v: A \rightarrow E \times F$ to be either an isomorphism of abelian surfaces, or an étale cyclic cover of degree $d_{v}=2,3$ (as in the hypotheses of Theorem 4.4). Finally we assume that $p>\operatorname{deg} v$.

Remark 4.6 Since the exponent of an isogeny divides its degree, the exponent of the isogeny $v$ of Setting 4.5 is either 1 if $v$ is an isomorphism, or $d_{v}$ otherwise. Now let $\mu: E \times F \rightarrow A$ be an isogeny of exponent $d_{v}$ such that $\mu \circ v=\left(d_{v}\right)_{A}$. Then the dual isogeny $\widehat{\mu}: \widehat{A} \rightarrow E \times F$ is either an isomorphism, or else its degree and exponent satisfy $(\operatorname{deg} \widehat{\mu}, \exp \widehat{\mu})=\left(d_{v}^{3}, d_{v}\right)$.

As an application of Theorem 2.9 we deduce that both the isogenies $v$ and $\widehat{\mu}$ of Setting 4.5 and Remark 4.6 lift to the ring of Witt vectors. In the following result we check that their degrees and exponents remain unchanged when passing from the special fiber to the general fiber. 
Proposition 4.7 Let $E$ and $F$ be elliptic curves and $\varphi: A \rightarrow E \times F$ be an étale isogeny of abelian surfaces with degree relatively prime with $p$. If $\mathcal{E} \rightarrow R$ and $\mathcal{F} \rightarrow R$ are projective lifts of $\mathcal{E}$ and $\mathcal{F}$ over an Henselian local ring $R$ with residue field $k$, then there exists a projective lift $\mathcal{A} \rightarrow R$ of $A$ and an isogeny $\varphi_{R}: \mathcal{A} \rightarrow \mathcal{E} \times_{R} \mathcal{F}$ such that $\varphi_{R}$ lifts $\varphi$ and its restriction $\varphi_{\eta}: A_{\eta} \rightarrow E_{\eta} \times F_{\eta}$ to the geometric general fibers is an isogeny with $\operatorname{deg} \varphi_{\eta}=\operatorname{deg} \varphi$ and $\exp \varphi_{\eta}=\exp \varphi$

Proof By Theorem 2.9 there is a projective lift $\mathcal{A} \rightarrow R$ of $A$ and an étale cover $\varphi_{R}: \mathcal{A} \rightarrow$ $\mathcal{E} \times{ }_{R} \mathcal{F}$ that specializes to $\varphi$. Up to composing $\varphi_{R}$ with a translation of $\mathcal{E} \times{ }_{R} \mathcal{F}$, we can suppose that $\varphi_{R}$ is a homomorphism of groups. We are going to prove that the restriction of $\varphi_{R}$ to the geometric generic fiber of $\mathcal{A}$ is an isogeny $\varphi_{\eta}: A_{\eta} \rightarrow E_{\eta} \times F_{\eta}$ such that $\operatorname{deg} \varphi_{\eta}=\operatorname{deg} \varphi$. To see this we notice that the kernel $\mathcal{K}$ of $\varphi_{R}$ is a finite étale group over $R$ and moreover, as $\varphi$ is separable, we have

$$
\operatorname{deg} \varphi_{\eta}=\left|\operatorname{ker} \varphi_{\eta}\right|=\left|\mathcal{K}_{\eta}\right|=\left|\mathcal{K}_{k}\right|=|\operatorname{ker} \varphi|=\operatorname{deg} \varphi
$$

where $\mathcal{K}_{k}$ is the closed fiber and $\mathcal{K}_{\eta}$ is the geometric generic fiber. In addition, since $\mathcal{K}$ is a finite étale group scheme over $R$, the closed fiber is killed by multiplication by $n$ if, and only if, the same is true for the geometric generic fiber. So we have also equality of the exponents.

We will deduce Theorem 4.4 from the following technical proposition.

Proposition 4.8 Assume the hypotheses of Setting 4.5 and let $\Phi: \mathbf{D}(B) \rightarrow \mathbf{D}(A)$ be an equivalence of derived categories of abelian surfaces. Suppose that there exists an equivalence $\Psi: \mathbf{D}(B) \rightarrow \mathbf{D}(C)$ with $C \in\{A, \widehat{A}\}$ such that the Mukai vector

$$
v:=(r, l, \chi)=\Psi^{\mathrm{CH}}(0,0,1)
$$

satisfies the following conditions:

$\left(E_{1}\right) r$ is positive;

(E) the class $l \in \mathrm{CH}^{1}(C)$ is is ample;

$\left(E_{3}\right) \chi$ is coprime with $r$.

Set $\lambda=v$ if $C=A$, and $\lambda=\widehat{\mu}$ otherwise (see Remark 4.6). In addition assume that there exist projective lifts $\mathcal{E} \rightarrow W$ and $\mathcal{F} \rightarrow W$ of $E$ and $F$ over a finite ramified extension of the ring of Witt vectors respectively such that the following conditions hold:

$\left(A_{1}\right)$ If we let $\lambda_{W}: \mathcal{C} \rightarrow \mathcal{E} \times_{W} \mathcal{F}$ be the lift of $\lambda$ determined by Proposition 4.7 , and let $L$ an ample line bundle on $C$ with numerical class $l$, then $L$ lies in the image of the restriction map

$$
\rho: \operatorname{Pic}(\mathcal{C}) \rightarrow \operatorname{Pic}(C) .
$$

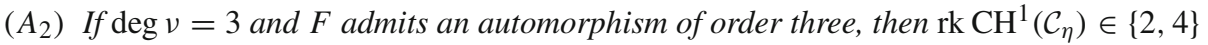
where $\mathcal{C}_{\eta}$ is the geometric generic fiber of $\mathcal{C} \rightarrow W$.

Then $B$ is either isomorphic to $C$ or $\widehat{C}$. In particular we have that $\operatorname{FM}(A)$ is trivial.

Proof We divide our argument in several steps.

Step 1. We first prove that there exists a projective lift $\mathcal{B} \rightarrow W$ of $B$ such that the geometric generic fiber $\mathcal{B}_{\eta}$ is derived equivalent to $\mathcal{C}_{\eta}$. Let $L$ be an ample line bundle on $C$ with class $l$. Then Theorem 3.3 implies that $B$ is isomorphic to a moduli space $\mathcal{M}_{l}(v)$ of Gieseker-stable 
shaves with Mukai vector $v=(r, l, \chi) \in \mathrm{CH}^{*}(C)$. Now consider a preimage $\widetilde{L}$ of $L$ under $\rho$ as in $\left(A_{1}\right)$ and the relative moduli space

$$
\mathcal{M}_{\tilde{l}}(\widetilde{v}) \rightarrow W
$$

where $\widetilde{v}_{l}:=(r, \tilde{l}, \chi)$ and $\tilde{l}$ is the class of $\widetilde{L}$. As discussed in Sect. 2.7, this is a projective lift of $B$ and the geometric generic fiber $\mathcal{M}_{\eta}$ is a moduli space of Gieseker-stable sheaves on $\mathcal{C}_{\eta}$ with Mukai vector $v_{\eta}=\left(r, \widetilde{l}_{\mid \mathcal{C}_{\eta}}, \chi\right)$. Therefore as discussed in Theorem 2.11, the conditions (E1), (E3), and $\left\langle v_{\eta}, v_{\eta}\right\rangle_{\mathcal{C}_{\eta}}=0$ imply that $\mathcal{M}_{\eta}$ is an abelian surface. In addition there exists a universal family $\mathcal{U}_{\eta}$ on $\mathcal{M}_{\eta} \times \mathcal{C}_{\eta}$ that induces an equivalence $\Phi_{\mathcal{U}}: \mathbf{D}\left(\mathcal{C}_{\eta}\right) \rightarrow \mathbf{D}\left(\mathcal{M}_{\eta}\right)$.

Step 2. Now we prove that under the assumptions of Theorem 4.4 the abelian surface $\mathcal{C}_{\eta}$ is isomorphic to either $\mathcal{M}_{\eta}$ or its dual $\widehat{\mathcal{M}_{\eta}}$. By the Lefschetz principle we can suppose that the abelian surface $C$ is defined over a subfield of the complex numbers $\mathbf{C}$ and therefore that $\mathcal{C}_{\eta}$ is defined over $\mathbf{C}$. Suppose first that $v: A \rightarrow E \times F$ is an isomorphism. Then both $\lambda$ and $\lambda_{W}$ are isomorphisms and therefore so is the restriction $\lambda_{\eta}: \mathcal{C}_{\eta} \rightarrow \mathcal{E}_{\eta} \times \mathcal{F}_{\eta}$ of $\lambda_{W}$ to the geometric generic fibers. As a product of elliptic curves has no non-trivial Fourier-Mukai partners (Theorem 4.1), there is an isomorphism $\mathcal{C}_{\eta} \simeq \mathcal{M}_{\eta}$.

Suppose now that $v: A \rightarrow E \times F$ is a degree two cyclic cover. By Remark 4.6 we have $(\operatorname{deg} \lambda, \exp \lambda) \in\{(2,2),(8,2)\}$ and by Proposition 4.7 we have $\left(\operatorname{deg} \lambda_{\eta}, \exp \lambda_{\eta}\right)=$ $(\operatorname{deg} \lambda, \exp \lambda)$. Therefore by Propositions 4.2 and 4.3 we deduce that either $\mathcal{C}_{\eta} \simeq \mathcal{M}_{\eta}$ or $\mathcal{C}_{\eta} \simeq \widehat{\mathcal{M}_{\eta}}$. The case when $v$ has degree three follows similarly by using the condition $\left(A_{2}\right)$. Step 3. The argument of [17, Lemma 6.5] (based on a result of Matsusaka-Mumford) proves that the isomorphism $\mathcal{C}_{\eta} \simeq \mathcal{M}_{\eta}$ (resp. $\mathcal{C}_{\eta} \simeq \widehat{\mathcal{M}_{\eta}}$ ) between the geometric generic fibers of the two liftings induces an isomorphism $C \simeq B$ (resp. $C \simeq \widehat{B}$ ) between the closed fibers. This immediately yields that either $B \simeq A$ or $B \simeq \widehat{A}$, and hence that $\operatorname{FM}(A)$ is trivial.

\section{Finding a suitable equivalence}

In this section we finish the proof of Theorem 1.1. According to Proposition 4.8, we only need to verify its hypotheses. We work under the hypotheses of Setting 4.5 and assume that the abelian surface $B$ is a Fourier-Mukai partner of $A$. In the following we will examine two cases: $(a)$ at least one of the two elliptic curves $E$ or $F$ is ordinary, and $(b)$ both $E$ and $F$ are supersingular.

The following two propositions show the existence of an equivalence $\Psi: \mathbf{D}(B) \rightarrow \mathbf{D}(C)$ satisfying the hypotheses $\left(E_{1}\right),\left(E_{2}\right)$ and $\left(E_{3}\right)$ of Proposition 4.8 , without further assumptions on the elliptic curves.

Proposition 5.1 Let $A$ and $B$ be abelian surfaces and $\Phi: \mathbf{D}(B) \rightarrow \mathbf{D}(A)$ be an equivalence of derived categories. Fix two distinct primes $p_{1}$ and $p_{2}$. Then there exists an equivalence $\Psi: \mathbf{D}(B) \rightarrow \mathbf{D}(C)$ with $C \in\{A, \widehat{A}\}$ such that the vector

$$
(r, l, \chi):=\Psi^{\mathrm{CH}}(0,0,1)
$$

satisfies one the two following statements:

(i) $r$ is relatively prime with both $p_{1}$ and $p_{2}$;

(ii) either $p_{1}$ divides $r$ but not $\chi$ and $p_{2}$ divides $\chi$ but not $r$, or vice versa. 
Proof Set

$$
\begin{aligned}
& v_{0}:=\left(r_{0}, l_{0}, \chi_{0}\right)=\Phi^{\mathrm{CH}}(0,0,1) \\
& w_{0}:=\left(s_{0}, h_{0}, \xi_{0}\right)=\Phi^{\mathrm{CH}}(1,0,0) .
\end{aligned}
$$

Since $\Phi$ is an equivalence, by (2.2) we have that

$$
1=\left\langle v_{0}, w_{0}\right\rangle_{A}=I-s_{0} \chi_{0}-r_{0} \xi_{0}, \quad \text { where } \quad I:=l_{0} \cdot h_{0} .
$$

Let $H_{0}$ be a line bundle on $A$ such that its class in the Néron-Severi group is $h_{0}$. Therefore at the level of numerical Chow rings the equivalence $\Phi_{n}:=T_{A}\left(H_{0}^{\otimes n}\right) \circ \Phi: \mathbf{D}(B) \rightarrow \mathbf{D}(A)$ $\left(n \in \mathbf{Z}_{>0}\right)$ sends $(0,0,1)$ to

$$
v_{n}:=\Phi_{n}^{\mathrm{CH}}(0,0,1)=\left(r_{0}, l_{0}+r_{0} n h_{0}, \chi_{n}\right)
$$

where

$$
\chi_{n}:=\chi_{0}+n I+r_{0} n^{2} \frac{h_{0}^{2}}{2} .
$$

We divide the proof in five cases.

Case I Suppose that neither $p_{1}$ nor $p_{2}$ divides $\chi_{0}$. In this case the equivalence $\Psi$ is given by the composition $\mathcal{S}_{A} \circ \Phi: \mathbf{D}(B) \rightarrow \mathbf{D}(\widehat{A})$.

Case II Suppose that both $p_{1}$ and $p_{2}$ divide both $r_{0}$ and $\chi_{0}$. By (5.1) we see that $I$ is relatively prime with $p_{1}$ and $p_{2}$ as well. Now choose a positive integer $n$ coprime with both $p_{1}$ and $p_{2}$. Therefore by looking at the definition (5.2) of $\chi_{n}$, this immediately implies that $\chi_{n}$ is relatively prime to both $p_{1}$ and $p_{2}$. We conclude then as in Case I.

Case III Suppose that both $p_{1}$ and $p_{2}$ divide $r_{0}$, and that precisely one of them, say $p_{1}$, divides $\chi_{0}$. We choose a positive integer $n$ such that $n$ is relatively prime to both $p_{1}$ and $p_{2}$. By (5.1) $I$ is relatively prime to $p_{1}$, and by (5.2) $p_{1}$ does not divide $\chi_{n}$. Moreover, again by (5.2) and the fact that $n$ is general, we can suppose that $p_{2}$ does not divide $\chi_{n}$ as well. We then set $\Psi:=\mathcal{S}_{A} \circ \Phi_{n}$.

Case IV Suppose that both $p_{1}$ and $p_{2}$ divide $\chi_{0}$ and that precisely one of them, say $p_{1}$, divides $r_{0}$. In this case we proceed as in Case III by considering the composition $\mathcal{S}_{A} \circ \Phi$ in place of $\Phi$.

Case V Suppose that one of the primes, say $p_{1}$, divides both $r_{0}$ and $\chi_{0}$, but $p_{2}$ divides neither $r_{0}$ nor $\chi_{0}$. Let $n=p_{2}$ and consider $\Phi_{n}$. By (5.1) $p_{1}$ does not divide $I$, and hence $p_{1}$ does not divide $\chi_{n}$. Moreover, by our choice of $n$, we have that $p_{2}$ does not divide $\chi_{n}$ as well. We then conclude as in Case I.

Proposition 5.2 Assume the hypotheses of Setting 4.5 and let $\Phi: \mathbf{D}(B) \rightarrow \mathbf{D}(A)$ be an equivalence of triangulated categories. Moreover assume that $\operatorname{deg} v \geq 2$. Then there exists an equivalence $\Xi: \mathbf{D}(B) \rightarrow \mathbf{D}(C)$ with $C \in\{A, \widehat{A}\}$ such that the vector

$$
(r, l, \chi):=\Xi^{\mathrm{CH}}(0,0,1)
$$

satisfies the following conditions:

(i) $r$ is positive and relatively prime with $p$;

(ii) the class $l \in \mathrm{CH}^{1}(C)$ is ample;

(iii) $\chi$ is relatively prime with $r$. 
Now set $\lambda=v$ if $C=A$ and $\lambda=\widehat{\mu}$ otherwise. Then the class $l$ is the pull-back of some ample class in $\mathrm{CH}^{1}(E \times F)$ via $\lambda$.

Proof By using Proposition 5.1 with $p_{1}=p$ and $p_{2}=\operatorname{deg} v$, we can find an equivalence $\Psi: \mathbf{D}(B) \rightarrow \mathbf{D}(C)$ with $C \in\{A, \widehat{A}\}$ such that the vector

$$
v_{0}:=\left(r_{0}, l_{0}, \chi_{0}\right)=\Psi^{\mathrm{CH}}(0,0,1)
$$

satisfies one of the two following conditions: $(a) r_{0}$ is relatively prime to both $p_{1}$ and $p_{2}$, or $(b)$ one of the primes $p_{1}$ and $p_{2}$ divides $r_{0}$ but not $\chi_{0}$, while the other divides $\chi_{0}$ but not $r_{0}$. Set $\lambda=v$ if $C=A$ and $\lambda=\widehat{\mu}$ otherwise. We claim that there exists an equivalence $\Xi_{1}: \mathbf{D}(B) \rightarrow \mathbf{D}(C)$ such that in the vector

$$
v_{1}:=\left(r_{1}, \lambda^{*} l_{1}, \chi_{1}\right)=\Xi_{1}^{\mathrm{CH}}(0,0,1),
$$

$r_{1}$ is positive and relatively prime with $p$, and $l_{1} \in \mathrm{CH}^{1}(E \times F)$. In order to prove the claim, we distinguish the two cases $(a)$ and $(b)$ mentioned above. First consider case $(a)$. As $r_{0}$ is not zero, we can make it positive by composing with the shift functor, if necessary. Let $L$ be a line bundle representing $l_{0}$ and $m$ be a positive integer such that $p_{2}^{2}$ divides $\left(r_{0} m+1\right)$. Hence we can write $1+r_{0} m=p_{2}^{2} u$ for some integer $u$ and we consider the composition $T_{C}\left(L^{\otimes m}\right) \circ \Psi$. Then

$$
\left(T_{C}\left(L^{\otimes m}\right) \circ \Psi\right)^{\mathrm{CH}}(0,0,1)=\left(r_{0},\left(p_{2}^{2} u\right) l_{0}, \chi_{1}\right)
$$

for some integer $\chi_{1}$. By Proposition 2.3 there exists a class $l_{1} \in \mathrm{CH}^{1}(E \times F)$ such that $\left(p_{2}^{2} u\right) l_{0}=\lambda^{*}\left(u l_{1}\right)$. This proves the claim in case $(a)$ as we can set $\Xi_{1}:=T_{C}\left(L^{\otimes m}\right) \circ \Psi$ and $v_{1}:=\left(r_{0},\left(p_{2}^{2} u\right) l_{0}, \chi_{1}\right)$.

Now consider case $(b)$. If necessary we replace $\Psi$ with $\mathcal{S}_{C} \circ \Psi$ in order to make $r_{0}$ divisible by $p_{1}=p$ but relatively prime with $p_{2}=\operatorname{deg} v$. Let $L$ and $m$ be as above. Then the equivalence $\Psi_{1}:=T_{C}\left(L^{\otimes m}\right) \circ \Psi$ sends the vector $(0,0,1)$ to

$$
\Psi_{1}^{\mathrm{CH}}(0,0,1)=\left(r_{0}, p_{2}^{2} u l_{0}, \chi_{0}+m l_{0}^{2}+r_{0} m^{2} \frac{l_{0}^{2}}{2}\right):=\left(r_{0}, p_{2}^{2} l_{2}, \chi_{2}\right)
$$

where $l_{2}$ is a class in $\mathrm{CH}^{1}(C)$. Since $\left\langle v_{0}, v_{0}\right\rangle_{C}=0$, we have that $l_{0}^{2}=2 r_{0} \chi_{0}$ and hence

$$
\chi_{2}=\chi_{0}+2 m r_{0} \chi_{0}+m^{2} r_{0}^{2} \chi_{0} .
$$

As $p_{1}$ divides $r_{0}$ but does not divide $\chi_{0}$, it follows that

$$
\chi_{2} \equiv \chi_{0} \not \equiv 0 \quad(\bmod p) .
$$

Hence,

$$
\left(\mathcal{S}_{C} \circ \Psi_{1}\right)^{\mathrm{CH}}(0,0,1)=\left(\chi_{2}, p_{2}^{2} \omega, r_{0}\right)=\left(\chi_{2}, \lambda^{*} \omega^{\prime}, r_{0}\right)
$$

for some elements $\omega \in \mathrm{CH}^{1}(C)$ and $\omega^{\prime} \in \mathrm{CH}^{1}(E \times F)$. Also, $p_{2}=\operatorname{deg} v$ does not divide $r_{0}$ (here we use the fact that $\mathcal{S}_{C}^{\mathrm{CH}}$ induces an homomorphism $\mathrm{CH}^{1}(C) \rightarrow \mathrm{CH}^{1}(\widehat{C})$; see Sect. 2.2). If necessary we can make the integer $\chi_{2}$ positive by composing with the shift functor. This concludes the proof of the claim.

Now let $w=(s, h, \xi)$ be the Mukai vector of $\Xi_{1}^{\mathrm{CH}}(1,0,0)$ so that $\left\langle w, v_{1}\right\rangle_{C}=1$. Moreover let $H$ be a line bundle whose numerical class is $h$ and note that

$$
I-r_{1} \xi-\chi_{1} s=1 \text { where } I:=l_{1} \cdot h .
$$


Choose a positive integer $n$ such that

$n p_{2}^{2} I+\chi_{1} \not \equiv 0 \quad(\bmod q) \quad$ for every prime divisor $q \neq p_{2}$ of $r_{1}$ that does not divide $I$.

Now set $\Xi_{2}:=T_{C}\left(H^{\otimes\left(n p_{2}^{2}\right)}\right) \circ \Xi_{1}$ and $\left(r_{2}, l_{2}, \chi_{2}\right):=\Xi_{2}^{\mathrm{CH}}(0,0,1)$. With a simple calculation we find

$$
r_{2}=r_{1}, \quad l_{2}=\lambda^{*} l_{1}+r_{1} n p_{2}^{2} h, \quad \chi_{2}=\chi_{1}+n p_{2}^{2} I+r_{1} n^{2} p_{2}^{4} \frac{h^{2}}{2} .
$$

We note that $r_{2}$ is not divisible by $p$, and moreover that by (5.4), $\chi_{2}$ is not divisible by any prime divisor $q \neq p_{2}$ of $r_{1}$ that does not divide $I$. On the other hand, if a prime divisor $q \neq p_{2}$ divides both $r_{1}$ and $I$, then by (5.3) it does not divide $\chi_{1}$, and hence does not divide $\chi_{2}$. Finally we prove that $\chi_{2}$ is not divisible by $p_{2}$ in the case where $p_{2}$ divides $r_{1}$. But this follows by the construction of $\Xi_{1}$, and by noting that in the case $(b)$ discussed earlier, $p_{2}$ does not divide $r_{0}$.

Now consider the composition $\Xi_{3}:=T_{C}\left(\lambda^{*} \Theta^{\otimes\left(r_{2} d\right)}\right) \circ \Xi_{2}$ where $\Theta:=\mathcal{O}_{E}\left(O_{E}\right) \otimes$ $\mathcal{O}_{F}\left(O_{F}\right)$ and $d \gg 0$ is a positive integer. By a direct computation we have that $\Xi_{3}^{\mathrm{CH}}$ sends the vector $(0,0,1)$ to

$$
v_{3}:=\left(r_{2}, l_{2}+r_{2}^{2} d \lambda^{*} \theta, \chi_{3}\right)
$$

where $\theta$ is the numerical class of $\Theta$ and

$$
\chi_{3}:=\chi_{2}+r_{2} d\left(l_{2} \cdot \lambda^{*} \theta\right)+r_{2}^{3} d^{2} \frac{\left(\lambda^{*} \theta\right)^{2}}{2} .
$$

By Proposition 2.3 and (5.4) we can write $l_{2}=\lambda^{*} l_{3}$ for some class $l_{3} \in \mathrm{CH}^{1}(E \times F)$, and hence

$$
\chi_{3} \equiv \chi_{2}+r_{2} d p_{2}^{c}\left(l_{3} \cdot \theta\right)+r_{2}^{3} p_{2}^{c} d^{2} \frac{\theta^{2}}{2} \equiv \chi_{2} \quad\left(\bmod p_{2}\right)
$$

as the isogeny $\lambda$ has degree either $p_{2}^{c}$ with $c=1$ if $C=A$, and $c=3$ otherwise. Therefore the first component of $v_{3}$ is still positive and relatively prime with $p$, while the second component is a pull-back of an ample class in $\mathrm{CH}^{1}(E \times F)$ (for $\left.d \gg 0\right)$. Moreover $\chi_{3} \equiv \chi_{2}\left(\bmod r_{2}\right)$ and hence it is still relatively prime with $r_{2}=r_{1}$. Our desired equivalence is hence given by $\Xi:=\Xi_{3}$.

Now we consider separately the case of supersingular abelian surfaces and the case of abelian surfaces that are not supersingular.

\subsection{The case where one of the two curves is ordinary}

The above results imply that when one of the elliptic curve involved is not supersingular then we can construct an equivalence satisfying $\left(E_{1}\right),\left(E_{2}\right)$ and $\left(E_{3}\right)$. In order to conclude we need to prove that the hypotheses $\left(A_{1}\right)$ and $\left(A_{2}\right)$ of Proposition 4.8 hold as well. We will first prove a couple of auxiliary results.

Proposition 5.3 Let $E$ and $F$ be ordinary elliptic curves. Then there exist projective liftings $\mathcal{E} \rightarrow W$ and $\mathcal{F} \rightarrow W$ of $E$ and $F$ over the ring of Witt vectors, respectively, such that the restriction morphism $\operatorname{Pic}\left(\mathcal{E} \times{ }_{W} \mathcal{F}\right) \rightarrow \operatorname{Pic}(E \times F)$ is surjective. 
Proof The product $E \times F$ is an ordinary abelian surface so it has a canonical lift $\left(\mathcal{Y} \rightarrow W, F_{\mathcal{Y}}\right)$ by virtue of Theorem 2.8. Moreover the restriction morphism $\operatorname{Pic}(\mathcal{Y}) \rightarrow \operatorname{Pic}(E \times F)$ is surjective. However by using the universal property of the fiber product, it is immediate to show that $\mathcal{Y} \simeq \mathcal{E} \times{ }_{W} \mathcal{F}$ where $\mathcal{E} \rightarrow W$ and $\mathcal{F} \rightarrow W$ are the canonical lifts of $E$ and $F$ respectively.

Proposition 5.4 If $E$ is an ordinary elliptic curve and $F$ is supersingular, then for any projective lifts $\mathcal{E} \rightarrow W$ and $\mathcal{F} \rightarrow W$ of $E$ and $F$ respectively, the restriction morphism $\operatorname{Pic}\left(\mathcal{E} \times{ }_{W} \mathcal{F}\right) \rightarrow \operatorname{Pic}(E \times F)$ is surjective.

Proof $\operatorname{As} \operatorname{Hom}(E, F)=0$ we obtain an isomorphism $\operatorname{Pic}(E \times F) \simeq \operatorname{Pic}(E) \times \operatorname{Pic}(F)$. Let now $\operatorname{pr}_{E}^{*} L_{E} \otimes \operatorname{pr}_{F}^{*} L_{F}$ be an arbitrary line bundle on $E \times F$. Since line bundles on curves lift, we can consider lifts $\mathcal{L}_{\mathcal{E}}$ and $\mathcal{L}_{\mathcal{F}}$ of $L_{E}$ and $L_{F}$ respectively. Hence the line bundle $\operatorname{pr}_{\mathcal{E}}^{*} \mathcal{L}_{\mathcal{E}} \otimes \operatorname{pr}_{\mathcal{F}}^{*} \mathcal{L}_{\mathcal{F}}$ on $\mathcal{E} \times_{W} \mathcal{F}$ is a lift of $\operatorname{pr}_{E}^{*} L_{E} \otimes \operatorname{pr}_{F}^{*} L_{F}$.

Proposition 5.5 Assume the hypotheses of Setting 4.5 and let $\Phi: \mathbf{D}(B) \rightarrow \mathbf{D}(A)$ be an equivalence of derived categories of abelian surfaces. If both $E$ and $F$ are ordinary elliptic curves, then the hypotheses of Proposition 4.8 hold.

Proof By Propositions 3.2 and 5.2 there exists an equivalence $\Xi: \mathbf{D}(B) \rightarrow \mathbf{D}(C)$ with $C \in\{A, \widehat{A}\}$ such that the vector $\Xi^{\mathrm{CH}}(0,0,1)=\left(r, \lambda^{*} l, \chi\right)$ satisfies the hypotheses $\left(E_{1}\right)$, $\left(E_{2}\right)$ and $\left(E_{3}\right)$ of Proposition 4.8. Let $L$ be a line bundle on $E \times F$ with class $l \in \mathrm{CH}^{1}(E \times F)$. By Proposition 5.3 there exist projective lifts $\mathcal{E} \rightarrow W$ and $\mathcal{F} \rightarrow W$ of $E$ and $F$ to the ring of Witt vectors $W$, and a lift $\mathcal{L} \in \operatorname{Pic}\left(\mathcal{E} \times{ }_{W} \mathcal{F}\right)$ of $L$. Let $\lambda_{W}: \mathcal{C} \rightarrow \mathcal{E} \times_{W} \mathcal{F}$ be the lift of $\lambda: C \rightarrow E \times F$ defined by Proposition 4.7. It follows that $\lambda_{W}^{*} \mathcal{L}$ lifts $\lambda^{*} L$ which proves condition $\left(A_{1}\right)$. In order to prove $\left(A_{2}\right)$ we can assume that $F$ has an automorphism of order three. Then by Theorem 2.8 the geometric generic fiber $\mathcal{F}_{\eta}$ admits an automorphism of order three and hence $\operatorname{rkCH}^{1}\left(\mathcal{E}_{\eta} \times \mathcal{F}_{\eta}\right) \in\{2,4\}$ where $\mathcal{E}_{\eta}$ is the geometric generic fiber of the lift $\mathcal{E} \rightarrow W$.

Proposition 5.6 Assume the hypotheses of Setting 4.5 and let $\Phi: \mathbf{D}(B) \rightarrow \mathbf{D}(A)$ be an equivalence of derived categories of abelian surfaces. If $E$ is ordinary and $F$ is supersingular or vice versa, then the hypotheses of Proposition 4.8 hold.

Proof By Propositions 3.2 and 5.2 there exists an equivalence $\Xi: \mathbf{D}(B) \rightarrow \mathbf{D}(C)$ with $C \in$ $\{A, \widehat{A}\}$ such that the vector $\Xi^{\mathrm{CH}}(0,0,1)=\left(r, \lambda^{*} l, \chi\right)$ satisfies the hypotheses $\left(E_{1}\right),\left(E_{2}\right)$ and $\left(E_{3}\right)$ of Proposition 4.8. By Proposition 5.4 we can choose general lifts $\mathcal{E}$ and $\mathcal{F}$ of $E$ and $F$ to $W$ respectively and a line bundle $\mathcal{L} \in \operatorname{Pic}(\mathcal{C})$ such that the condition $\left(A_{1}\right)$ holds (see the argument of Proposition 5.5). Furthermore, since any fixed elliptic curve has a 1-dimensional family of deformations and for any choice of $\mathcal{E}$, there are only finitely many elliptic curves isogenous to $\mathcal{E}_{\eta}$, we may choose $\mathcal{E}$ and $\mathcal{F}$ so that there are no non-trivial morphisms between the geometric generic fibers $\mathcal{E}_{\eta}$ and $\mathcal{F}_{\eta}$. It follows that $\operatorname{rk} \operatorname{NS}\left(\mathcal{E}_{\eta} \times \mathcal{F}_{\eta}\right)=2$ independently of whether $F$ admits an automorphism group of order three or not. As in Proposition 5.5 this immediately implies that $\operatorname{rk} \operatorname{NS}\left(\mathcal{C}_{\eta}\right)=2$.

\subsection{Supersingular case}

We are going to prove that the hypotheses of Proposition 4.8 hold when the elliptic curves $E$ and $F$ are both supersingular. The following proposition proves the conditions $\left(E_{1}\right),\left(E_{2}\right)$ and $\left(E_{3}\right)$. 
Proposition 5.7 Assume the hypotheses of Setting 4.5 with $\operatorname{deg} v \geq 2$ and assume that the elliptic curves $E$ and $F$ are supersingular. Moreover let $\Phi: \mathbf{D}(B) \rightarrow \mathbf{D}(A)$ be an equivalence of derived categories of abelian surfaces. Then there exists an equivalence $\Xi: \mathbf{D}(B) \rightarrow \mathbf{D}(C)$ with $C \in\{A, \widehat{A}\}$ such that the Mukai vector

$$
(r, l, \chi):=\Xi^{\mathrm{CH}}(0,0,1)
$$

satisfies the following conditions:

(i) $r$ is positive and relatively prime with $p$;

(ii) the class $l \in N S(C)$ is ample;

(iii) $\chi$ is relatively prime with $r$.

Now set $\lambda=v$ if $C=A$, and $\lambda=\widehat{\mu}$ otherwise. Then $l=\lambda^{*} l^{\prime}$ where $l^{\prime}=l\left(\varphi, d_{1}, d_{2}\right)$ is the class of a line bundle on $E \times F$ with $\varphi$ an étale isogeny.

Proof Proceeding as in the proof of Proposition 5.2, we can construct an equivalence $\Psi_{1}$ : $\mathbf{D}(B) \rightarrow \mathbf{D}(C)$ where $C \in\{A, \widehat{A}\}$ such that in the vector

$$
v_{1}:=\left(r_{1}, \lambda^{*} l_{1}, \chi_{1}\right)=\Psi_{1}^{\mathrm{CH}}(0,0,1),
$$

$r_{1}$ is relatively prime with $p$, and $l_{1}=l\left(\varphi, m_{1}, m_{2}\right)$ is the class of a line bundle on $E \times F$ for some morphism $\varphi: F \rightarrow E$ and integers $m_{1}, m_{2}$ (see Proposition 2.7). We notice that we can assume $r_{1}$ positive by eventually composing with the shift functor. First, suppose that $\varphi$ is either the constant morphism or non-separable. Let $t \neq p$ be a prime and let $\xi: F \rightarrow E$ be a separable isogeny of degree $t^{k}\left(k \in \mathbf{Z}_{>0}\right)$ determined by Proposition 2.4. Now consider the composition of equivalences $\Psi_{2}:=T_{C}\left(\lambda^{*}\left(1_{E} \times \xi\right)^{*} \mathcal{M}_{E}\right) \circ \Psi_{1}: \mathbf{D}(B) \rightarrow \mathbf{D}(C)$. As the class of $\left(1_{E} \times \xi\right)^{*} \mathcal{M}_{E}$ is $l(\xi, 0,0)$, by Corollary 2.6 we have that $\Psi_{2}^{\mathrm{CH}}$ sends the vector $(0,0,1)$ to

$$
v_{2}:=\left(r_{1}, \lambda^{*} l\left(\varphi+r_{1} \xi, m_{1}, m_{2}\right), \chi_{2}\right)
$$

for some integer $\chi_{2}$. Recall that if $f_{1}$ and $f_{2}$ are two isogenies of elliptic curves with $f_{1}$ inseparable, then $f_{1}+f_{2}$ is separable if and only if $f_{2}$ is separable. Then as $r_{1}$ is relatively prime with $p$, the isogeny $\gamma:=\varphi+r_{2} \xi$ is separable (indeed the composition of separable isogenies is separable, and the multiplication-by- $n$ map is separable if and only if $n$ is coprime with $p$ ), and hence étale.

Let $w:=(s, h, \zeta)=\Psi_{2}^{\mathrm{CH}}(1,0,0)$ and note that $\left\langle v_{2}, w\right\rangle_{C}=1$. By Proposition 2.3 the class $p_{2}^{2} h$ (where $p_{2}=\operatorname{deg} v$ ) is in the image of the pull-back $\lambda^{*}: \mathrm{CH}^{1}(E \times F) \rightarrow \mathrm{CH}^{1}(C)$. Therefore we can write

$$
p_{2}^{2} h=\lambda^{*} l\left(\psi, k_{1}, k_{2}\right)
$$

for some morphism $\psi$ and integers $k_{1}, k_{2}$. Consider the equivalence $\Psi_{3}:=T_{C}\left(H^{\otimes\left(n p p_{2}^{2}\right)}\right) \circ$ $\Psi_{2}$ which sends the vector $(0,0,1)$ to

$$
\begin{aligned}
v_{3}:= & \left(r_{1}, \lambda^{*} l\left(\gamma+r_{1} p n \psi, m_{1}+r_{1} p n k_{1}, m_{2}+r_{1} p n k_{2}\right),\right. \\
& \left.\chi_{2}+p p_{2}^{2} n I+r_{1} p^{2} p_{2}^{4} n^{2} \frac{h^{2}}{2}\right)
\end{aligned}
$$

where

$$
I:=\lambda^{*}\left(\gamma, m_{1}, m_{2}\right) \cdot h
$$


As $\left(r_{1} p n\right) \psi$ is either the zero morphism or non-separable, we have that $\gamma+\left(r_{1} p n\right) \psi$ is a separable isogeny. Now choose a positive integer $n$ so that

$p p_{2}^{2} n I+\chi_{2} \not \equiv 0 \quad(\bmod q) \quad$ for every prime divisor $q \neq p_{2}$ of $r_{2}$ that does not divide $I$.

By the same argument as Proposition 5.2, this choice of $n$ ensures that the third component of $v_{3}$ is relatively prime with $r_{1}$.

Now we define the line bundle $\Theta:=\mathcal{O}_{E}\left(O_{E}\right) \otimes \mathcal{O}_{F}\left(O_{F}\right)$ on $E \times F$ and we consider the equivalence $T_{C}\left(\lambda^{*} \Theta^{\otimes\left(r_{1} d\right)}\right) \circ \Psi_{3}$ with $d \gg 0$ a positive integer. Take $\theta:=l\left(0_{E}, 1,1\right)$ to be the class of $\Theta$. The equivalence $T_{C}\left(\lambda^{*} \Theta^{\otimes\left(r_{1} d\right)}\right) \circ \Psi_{3}$ sends $(0,0,1)$ to

$$
v_{4}=\left(r_{1}, \lambda^{*}\left(\gamma+r_{1} n p \psi, m_{1}+r_{1} p n k_{1}+r_{1}^{2} d, m_{2}+r_{2} p n k_{2}+r_{1}^{2} d\right), \chi_{4}\right)
$$

where

$$
\begin{aligned}
\chi_{4}:= & \chi_{2}+p p_{2}^{2} n I+r_{1} p^{2} p_{2}^{4} n^{2} \frac{h^{2}}{2} \\
& +r_{1} d\left(\theta \cdot \lambda^{*} l\left(\gamma+r_{1} p n \psi, m_{1}+r_{1} p n k_{1}, m_{2}+r_{1} p n k_{2}\right)\right)+r_{1}^{3} d^{2} \frac{\theta^{2}}{2} .
\end{aligned}
$$

As $\chi_{4}$ is congruent to the third component of $v_{3}$ modulo $r_{1}$, we have that $\chi_{4}$ is still relatively prime with $r_{1}$. Moreover, for $d$ sufficiently large the second component of $v_{4}$ is ample. Finally, $r_{1}$ is relatively prime with $p$, and hence $T_{C}\left(\lambda^{*} \Theta^{\otimes\left(r_{1} d\right)}\right) \circ \Psi_{3}$ is the equivalence we are looking for.

To conclude the proof we need to analyze the case when $\varphi$ is separable. But in this case the proof is simpler as there is no need to introduce the isogeny $\xi$ and the equivalence $\Psi_{2}$. Then it is enough to set $\gamma=\varphi$ and proceed as in the inseparable case.

Proposition 5.8 Assume the hypotheses of Setting 4.5 and let $\Phi: \mathbf{D}(B) \rightarrow \mathbf{D}(A)$ be an equivalence of derived categories of abelian surfaces. Moreover assume that the elliptic curves $E$ and $F$ are both supersingular. Then the hypotheses of Proposition 4.8 hold.

Proof By Propositions 3.2 and 5.7 we can find an equivalence $\Xi: \mathbf{D}(B) \rightarrow \mathbf{D}(C)$ with $C \in\{A, \widehat{A}\}$ such that the vector

$$
v:=\left(r, \lambda^{*} l\left(\varphi, m_{1}, m_{2}\right), \chi\right)=\Xi^{\mathrm{CH}}(0,0,1)
$$

satisfies the assumptions $\left(E_{1}\right),\left(E_{2}\right)$, and $\left(E_{3}\right)$ of Proposition 4.8. The class $l\left(\varphi, m_{1}, m_{2}\right)$ is the class of an ample line bundle $L\left(\varphi, M_{1}, M_{2}\right)$ on $E \times F$ where $M_{1}$ and $M_{2}$ are line bundles on $E$ and $F$ respectively, and $\varphi: F \rightarrow E$ is the constant morphism $O_{E}$ in the case where $v$ is an isomorphism, or an étale isogeny otherwise. Let $\mathcal{E} \rightarrow W$ be a lift of $E$ to the ring of Witt vectors. If $\varphi=O_{E}$ is the constant morphism, then on any lift $\mathcal{F} \rightarrow W$ of $F$ we can construct a lift of $L=M_{1} \otimes M_{2}$ by simply lifting $M_{1}$ and $M_{2}$ to $W$ and taking their exterior product. Now assume that $\varphi$ is a separable isogeny. Then Theorem 2.9 determines a projective lift $\mathcal{F} \rightarrow W$ of $\mathcal{F}$ and an étale cover $\tilde{\varphi}: \mathcal{F} \rightarrow \mathcal{E}$ that specializes to $\varphi$. It is not difficult to check that $L\left(\varphi, M_{1}, M_{2}\right)$ lifts to a line bundle $\mathcal{L}$ on $\mathcal{E} \times_{W} \mathcal{F}$. This goes as follows. As line bundles on curves lift, we can find a lift of $\mathcal{O}_{E}\left(O_{E}\right)$ which in turn allows us to construct a lift $\mathcal{M}_{E, W}$ to $W$ of the Mumford line bundle $\mathcal{M}_{E}$ ( $c f$. Sect. 2.4). Denote by $M_{1, W}$ and $M_{2, W}$ the liftings of $M_{1}$ and $M_{2}$ on $\mathcal{E}$ and $\mathcal{F}$ respectively. The line bundle $\mathcal{L}:=\left(1_{\mathcal{E}} \times_{W} \widetilde{\varphi}\right)^{*} \mathcal{M}_{E, W} \otimes \operatorname{pr}_{\mathcal{E}}^{*} M_{1, W} \otimes \operatorname{pr}_{\mathcal{F}}^{*} M_{2, W}$ is a lift of $L\left(\varphi, M_{1}, M_{2}\right)$, where $\operatorname{pr}_{\mathcal{E}}$ and $\mathrm{pr}_{\mathcal{F}}$ are the obvious projections. In conclusion the line bundle $\lambda_{W}^{*} \mathcal{L}$ lifts $\lambda^{*} L\left(\varphi, M_{1}, M_{2}\right)$ where $\lambda_{W}: \mathcal{C} \rightarrow \mathcal{E} \times{ }_{W} \mathcal{F}$ is the lift from Proposition 4.8. This proves the condition $\left(A_{1}\right)$ in the case $\operatorname{deg} v=1,2$. 
Now suppose $\operatorname{deg} v=3$ and that $F$ has an automorphism $\rho: F \rightarrow F$ of order three. By a result of Deuring ( $c f$. [29, p. 189 or p. 172]) it is possible to lift a supersingular elliptic curve together with an endomorphism over a finite ramified extension $W$ of the ring of Witt vectors. Thus we have lifts $\mathcal{F}_{0} \rightarrow W$ and $\rho_{W}: \mathcal{F}_{0} \rightarrow \mathcal{F}_{0}$ of $F$ and $\rho$ to $W$. The étale cover $\widehat{\varphi}: E \rightarrow F$ determines a lift $\mathcal{E}_{0}$ of $E$ together with a lift $\widetilde{\varphi}_{0}$ of $\widehat{\varphi}$ over $W$. Now, denote by $\lambda_{W, 0}: \mathcal{C}_{0} \rightarrow \mathcal{E}_{0} \times{ }_{W} \mathcal{F}_{0}$ the lift of $\lambda$ as defined in Proposition 4.8. After noting that there is an isomorphism $\left(1_{E} \times \varphi\right)^{*} \mathcal{M}_{E} \simeq\left(\widehat{\varphi} \times 1_{F}\right)^{*} \mathcal{M}_{F}$, and hence that

$$
L\left(\varphi, M_{1}, M_{2}\right) \simeq\left(\widehat{\varphi} \times 1_{F}\right)^{*} \mathcal{M}_{F} \otimes \operatorname{pr}_{E}^{*} M_{1} \otimes \operatorname{pr}_{F}^{*} M_{2},
$$

the previous argument shows that the line bundle $\lambda^{*} L\left(\varphi, M_{1}, M_{2}\right)$ lifts to $\mathcal{C}_{0}$. Moreover the restriction of $\rho_{W}$ to the geometric generic fiber $\left(\mathcal{F}_{0}\right)_{\eta}$ is an automorphism that is not proportional to the identity. Hence $\mathrm{rk} \mathrm{CH}^{1}\left(\left(\mathcal{E}_{0}\right)_{\eta} \times\left(\mathcal{F}_{0}\right)_{\eta}\right)$ is either 2 or 4 according to whether or not there is an isogeny between $\left(\mathcal{E}_{0}\right)_{\eta}$ and $\left(\mathcal{F}_{0}\right)_{\eta}$. As the rank of the Néron-Severi group does not change under separable isogenies, we have that $\operatorname{rk~}^{1}\left(\left(\mathcal{C}_{0}\right)_{\eta}\right) \in\{2,4\}$ where $\left(\mathcal{C}_{0}\right)_{\eta}$ is the geometric generic fiber of $\mathcal{C}_{0}$. In particular the assumption $\left(A_{2}\right)$ holds.

\section{Canonical covers of Enriques surfaces}

An Enriques surface $S$ over an algebraically closed field $k$ of characteristic $p \neq 2$ is a smooth projective minimal surface with canonical bundle $\omega_{S}$ of order two, $\chi\left(\mathcal{O}_{S}\right)=1$, and $b_{2}(S)=10$. The canonical cover $\pi: X \rightarrow S$ induced by $\omega_{S}$ is a double étale cover with $X$ a $K 3$ surface. Let $\Phi: \mathbf{D}(Y) \rightarrow \mathbf{D}(X)$ be a derived equivalence so that $Y$ is itself a $K 3$ surface by [17, Proposition 3.9]. We aim to prove Theorem 1.3, namely that $X \simeq Y$.

In [17] the authors prove that Shioda-supersingular $K 3$ surfaces do not admit any nontrivial Fourier-Mukai partners, thus we can assume that $X$ has Picard rank less than 22. Since in odd characteristic Shioda-supersingularity is equivalent to Artin-supersingularity ( $c f$. for instance [30]), we can assume that the formal Brauer group of $X$ is of finite height. In particular there exists a lift $\mathcal{X} \rightarrow W$ of $X$ over the ring of Witt vectors such that $\operatorname{NS}(X) \simeq \operatorname{NS}\left(\mathcal{X}_{\eta}\right)$, where as usual $\mathcal{X}_{\eta}$ is the geometric generic fiber [28, p. 505]. By the work of Jang [12, Theorem 2.5] there is a primitive embedding of the Enriques lattice $\Gamma(2):=U(2) \oplus E_{8}(-2)$ of $S$ into NS $(X)$ such that the orthogonal complement of the embedding does not contain any vector of self intersection -2 . Using the isomorphism $\mathrm{NS}(X) \simeq \mathrm{NS}\left(\mathcal{X}_{\eta}\right)$, together with the characterization of Enriques- $K 3$ surfaces in terms of its periods [27, Theorem 1.14], we deduce that $\mathcal{X}_{\eta}$ is a $K 3$ surface arising as the canonical cover of an Enriques surface. In particular $\mathcal{X}_{\eta}$ does not have any non-trivial Fourier-Mukai partner thanks to [34, Theorem 1.1]. On the other hand, [17, Proposition 8.2] implies that $Y$ is isomorphic to a moduli space $\mathcal{M}_{h}(v)$ of $h$-Gieseker-stable sheaves for some Mukai vector $v=(r, l, \chi)$. Since all the line bundles on $X$ deform to line bundles over $\mathcal{X} \rightarrow W$, we can consider the relative moduli space $\mathcal{M}_{\mathcal{X} / W}(r, \widetilde{l}, \chi) \rightarrow W$ where $\widetilde{l}$ is a lift of $l$. The geometric generic fiber of $\mathcal{M}_{\mathcal{X} / W}(r, \tilde{l}, \chi) \rightarrow W$ is by construction a Fourier-Mukai partner of $\mathcal{X}_{\eta}$ and therefore, by the previous considerations, it is isomorphic to $\mathcal{X}_{\eta}$. We conclude by using [17, Lemma 6.5], which says that the isomorphism between the geometric generic fibers of relative $K 3$ surfaces induces an isomorphism between the closed fibers.

Acknowledgements Open access funding provided by Stockholm University. This Project started when ST visited LL in the Summer of 2014 at the University of Bonn. LL and ST heartily thank Daniel Huybrechts for guiding them during the early stages of the Project and for his insights. Moreover LL and ST thank the Institute of Mathematics of the University of Bonn for the optimal working conditions offered. The authors all 
thank M. Olsson for useful advices and P. Sosna for carefully reading a first draft of this paper. Moreover KH thanks A. Bertram and E. Canton for helpful conversation; LL thanks A. Bellardini, C. Liedtke, C. Schnell and M. Talpo for comments and correspondence; ST thanks A. Bertram, C. Hacon, M. Lieblich, B. Moonen, K. Schwede, M. Talpo and B. Viray for very interesting mathematical conversation. She was also partially supported by the grant 261756 of the Research Councils of Norway. Finally the authors thank their previous institutions, where part of these project were ultimated, that respectively were: University of California at Berkeley and University of Utah, University of Bonn, Stonybrook University, and University of Florence, and the University of Utah. LL was partly supported by the SFB/TR45 "Periods, moduli spaces, and arithmetic of algebraic varieties" of the DFG (German Research Foundation). All authors are grateful to the unknown referee for carefully reading the paper, and for suggesting corrections to the discussion in Sect. 2.7 and a more direct proof (as well as the current more general statement) of Proposition 4.7.

Open Access This article is distributed under the terms of the Creative Commons Attribution 4.0 International License (http://creativecommons.org/licenses/by/4.0/), which permits unrestricted use, distribution, and reproduction in any medium, provided you give appropriate credit to the original author(s) and the source, provide a link to the Creative Commons license, and indicate if changes were made.

\section{References}

1. Badescu, L.: Algebraic Surfaces, Universitext. Springer, New York (2001)

2. Bartocci, C., Bruzzo, U., Hernández Ruipérez, D.: Fourier-Mukai and Nahm transforms in geometry and mathematical physics. In: Progress in Mathematics, vol. 276. Springer, Boston (2009)

3. Birkenhake, C., Lange, H.: Complex abelian varieties. In: Grundlehren der Mathematischen Wissenschaften, vol. 302, 2nd edn. Springer, Berlin (2004)

4. Bombieri, E., Mumumford, D.: Enriques' classification of surfaces in char. p. II. In: Complex Analysis and Algebraic Geometry, pp. 23-42. Iwanami Shoten, Tokyo (1977)

5. Bridgeland, T.: Fourier-Mukai transforms for surfaces and moduli spaces of stable sheaves. Ph.D. Thesis, University of Edinburgh (1998)

6. Bridgeland, T., Maciocia, A.: Complex surfaces with equivalent derived categories. Math. Z. 236(4), 677-697 (2001)

7. Huybrechts, D.: Fourier-Mukai Transform in Algebraic Geometry, Oxford Mathematical Monographs. Oxford University Press, Oxford (2006)

8. Huybrechts, D., Lehn, M.: The Geometry of Moduli Spaces of Sheaves, Cambridge Mathematical Library, Second edn. Cambridge University Press, Cambridge (2010)

9. Honigs, K.: Derived equivalent surfaces and abelian varieties, and their zeta functions. Proc. Am. Math. Soc. 143(10), 4161-4166 (2015)

10. Honigs, K.: Derived equivalent varieties and their Hasse-Weil Zeta functions. Ph.D. Thesis, University of California, Berkeley (2015)

11. Honigs, K.: Derived equivalence, Albanese varieties, and the zeta functions of 3-dimensional varieties. Proc. Am. Math. Soc. 146(3), 1005-1013 (2018)

12. Jang, J.: Néron-Severi group preserving lifting of K3 surfaces and applications. Math. Res. Lett. 22(3), 789-802 (2015)

13. Li, K.-Z., Oort, F.: Moduli of supersingular abelian varieties. In: Lecture Notes in Mathematics, vol. 1680. Springer, Berlin (1998)

14. Kohel, D.: Endomorphism ring of elliptic curves over finite fields. Ph.D. Thesis, University of California, Berkeley (1996)

15. Langer, A.: Semistable sheaves in positive characteristic. Ann. Math. (2) 159(1), 251-276 (2004)

16. Li, K.-Z., Oort, F.: Moduli of supersingular abelian varieties. In: Lecture Notes in Mathematics, vol. 1680. Springer, Berlin (1998)

17. Lieblich, M., Olsson, M.: Fourier-Mukai partners of K3 surfaces in positive characteristic. Ann. Sci. Éc. Norm. Supér. 48(5), 1001-1033 (2015)

18. Lieblich, M., Olsson, M.: A stronger derived Torelli theorem for $K 3$ surfaces. arXiv: 1512.06451

19. López Martín, A.C.: Fully faithfulness criteria for quasi-projective schemes. Collect. Math. 68(2), 219227 (2017)

20. Maruyama, M.: Construction of moduli spaces of stable sheaves via Simpson's idea. Moduli of vector bundles (Sanda, 1994; Kyoto, 1994). Lecture Notes in Pure and Appl. Math., vol. 179, pp. 147-187 (1996)

21. Milne, J.S.: Abelian varieties (v2.00) (2008). www.jmilne.org/math/ 
22. Mehta, V.B., Srinivas, V.: Varieties in positive characteristic with trivial tangent bundle, with an appendix by Srinivas and M. V. Nori. Compos. Math. 64(2), 191-212 (1987)

23. Mukai, S.: Duality between $\mathrm{D}(\mathrm{X})$ and $\mathrm{D}(\widehat{X})$ with its application to Picard sheaves. Nagoya Math. J. 81, 153-175 (1981)

24. Mukai, S.: Symplectic structure of the moduli space pf sheaves on an abelian or $K 3$ surface. Invent. Math. 77(2), 101-116 (1984)

25. Mukai, S.: On the moduli spaces of bundles on $K 3$ surfaces, I. Vector bundles on algebraic varieties (Bombay, 1984). Tata Inst. Fund. Res. Stud. Math. 11, 341-413 (1987)

26. Mumford, D.: Abelian varieties. Tata Institute of Fundamental Research Studies in Mathematics, vol. 5, Published for the Tata Inst., Bombay; by Hindustan Book Agency, New Delhi (2008)

27. Namikawa, Y.: Periods of Enriques surfaces. Math. Ann. 270(2), 201-222 (1985)

28. Nygaard, N., Ogus, A.: Tate's conjecture for K3 surfaces of finite height. Ann. Math. (2) 122, 461-507 (1985)

29. Oort, F.: Lifting algebraic curves, abelian varieties, and their endomorphisms to characteristic zero. In: Algebraic Geometry, Bowdoin, Proc. Sympos. Pure Math., vol. 46, pp. 165-195 (1985)

30. Pera, K.M.: The Tate conjecture for $K 3$ surfaces in odd characteristic. Invent. Math. 201(2), 625-668 (2015)

31. Serre, J.-P.: Local Fields. Graduate Graduate Texts in Mathematics, vol. 67. Springer, Berlin (2013)

32. Grothendieck, A.: Revêtements etales et Groupe Fondamental (SGA 1). In: LMN, vol. 224 (1971)

33. Silverman, J.: The Arithmetic of Elliptic Curves. GTM, vol. 106, 2nd edn. Springer, Dordrecht (2009)

34. Sosna, P.: Fourier-Mukai partners of canonical covers of bielliptic and Enriques surfaces. Rend. Semin. Mat. Univ. Padova 130, 203-213 (2013)

35. Ward, M.: Arithmetic properties of the derived category for Calabi-Yau varieties. Ph.D. Thesis, University of Washington (2014)

Publisher's Note Springer Nature remains neutral with regard to jurisdictional claims in published maps and institutional affiliations. 